\title{
Voltage Regulation Performance Evaluation of Distributed Energy Resource Management via Advanced Hardware-in-the-Loop Simulation ${ }^{+}$
}

\author{
Jing Wang, Harsha Padullaparti, Fei Ding, Murali Baggu * and Martha Symko-Davies
}

Citation: Wang, J.; Padullaparti, H.; Ding, F.; Baggu, M.; Symko-Davies, M. Voltage Regulation Performance Evaluation of Distributed Energy Resource Management via Advanced Hardware-in-the-Loop Simulation. Energies 2021, 14, 6734. https:// doi.org/10.3390/en14206734

Academic Editor: GM Shafiullah

Received: 4 August 2021

Accepted: 13 October 2021

Published: 16 October 2021

Publisher's Note: MDPI stays neutral with regard to jurisdictional claims in published maps and institutional affiliations.

Copyright: (c) 2021 by the authors. Licensee MDPI, Basel, Switzerland. This article is an open access article distributed under the terms and conditions of the Creative Commons Attribution (CC BY) license (https:// creativecommons.org/licenses/by/ $4.0 /)$.
National Renewable Energy Laboratory, Golden, CO 80401, USA; Jing.Wang@nrel.gov (J.W.); HarshaVardhana.Padullaparti@nrel.gov (H.P.); Fei.Ding@nrel.gov (F.D.);

Martha.Symko.Davies@nrel.gov (M.S.-D.)

* Correspondence: Murali.Baggu@nrel.gov

† This paper is an extended version of our paper published in 2020 IEEE Innovative Smart Grid Technologies (ISGT) North America, Washington, DC, USA, 17-20 February 2020.

\begin{abstract}
This paper evaluates the performance of coordinated control across advanced distribution management systems (ADMS), distributed energy resources (DERs), and distributed energy resource management systems (DERMS) using an advanced hardware-in-the-loop (HIL) platform. This platform provides a realistic laboratory testing environment, including accurate dynamic modeling of a real-world distribution system from a utility partner, real controllers (ADMS and DERMS), physical power hardware (DERs), and standard communications protocols. One grid servicevoltage regulation-is evaluated to show the performance of the coordinated grid automation system. The testing results demonstrate that the coordinated DERMS and ADMS system can effectively regulate system voltages within target operation limits using DERs. The realistic laboratory HIL testing results give utilities confidence in adopting the grid automation systems to manage DERs to achieve system-level control and operation objectives (e.g., voltage regulation). This helps utilities mitigate potential risks (e.g., instability) prior to field deployment.
\end{abstract}

Keywords: advanced distribution management system; distributed energy resource management system; hardware-in-the-loop; voltage regulation

\section{Introduction}

Power systems are evolving from centralized systems toward massively distributed energy systems with millions of controllable nodes [1]. Transformative changes, notably at the distribution level, are taking place where increasing numbers of distributed energy resources (DERs)—such as renewable generation devices, energy storage devices, and flexible loads-are becoming prominent considerations. This requires the grid planners and operators to modernize electric grids as uncontrolled/uncoordinated DERs may cause issues to the electricity grids, such as overvoltage problems. The utility industry is exploring ways to leverage DERs to enhance system operations, paving the way for distribution-level markets, and offer new services to customers [2].

Distributed energy resource management systems (DERMS) are emerging as scalable solutions to control and monitor groups of DERs at the grid edge [3]. DERMS will interface with distribution management systems (DMS) to provide the grid services as requested by the DMS [4]. New control strategies will be needed to manage DERs, DERMS, and DMS in a coherent manner to achieve system-level benefits, such as constraint management and DER flexibility utilization [5]. 
Various DERMS-related research has been developed and validated through numerical simulations. A hierarchical distributed DERMS algorithm was developed in [6] to solve optimal power flow (OPF) problems aiming to dispatch DERs for voltage regulation at minimal cost, and satisfactory performance was achieved with numerical simulation of a test feeder in OpenDSS. An OPF-based DERMS was developed and evaluated in [7] for congestion management (e.g., voltage profiles) and operational planning with historical advanced metering infrastructure (AMI) data in a simulation environment. An integrated DERMS for residential customers was developed in [8] to provide demand prediction, optimal planning, and real-time operation of DERs and the performance was evaluated in simulations with satisfactory results and effectiveness for DER management. An EMS for a smart community comprising residential units with DERs and a shared facility controller using noncooperative game theory was developed in [9] to minimize total operational cost for utility and electricity bills for customers, and a numerical study was performed to validate the effectiveness of the proposed DERMS control. A hierarchical, distributed, model predictive control-based energy management strategy for smart grids was developed in [10] comprising both centralized and distributed control approaches to solve and decompose the complex management objective function of large-scale power systems, and it was evaluated via both simulation and hardware experiments focusing not only on the control/optimization but also on communications problems. Work focusing on virtual power plant (VPP) applications for DERMS was developed in [11,12] that aimed to balance generation and load, handle emergent events in real-time, and optimize the benefits of DERs, especially energy storage, and validated through numerical simulations. An energy management system for hybrid AC/DC microgrid is developed in [13] to develop a risk-based uncertainty set optimization method to address the uncertainty of distributed energy resources and numerical simulation is performed to validate the design and prove the concept. A microgrid EMS system based on state machine is developed in [14] to manage transition operation of microgrid and optimal power flow in grid-connected mode and the concept is validated through numerical simulation.

All these works were validated, demonstrated, and tested using numerical simulations. Numerical simulations are a preliminary step toward proving the concept and showcasing the performance of DERMS for DER management and providing requested services to distribution grids; however, numerical simulations might not be reliable and sufficient because detailed models of the components are often unavailable or insufficient, the impact of physical communications systems and links are missing, and the dynamics and real responses of the hardware controller cannot be represented and evaluated. Therefore, further validation of DERMS is necessary to mitigate risks (e.g., instabilities, communications delay, malfunctioning of the components) prior to field deployment.

Hardware-in-the-loop (HIL) evaluation is the most effective approach to test DERMS because the DERMS hardware controller can dynamically interact with the distribution system model with real-time measurements and dispatched power set points and with hardware DER inverters through standard communication protocols as if the controller were interacting with real-world systems in the field [15]; thus, the stability of the whole system and the control performance of DERMS can be evaluated with much more reliable and trustworthy results. In particular, potential hazards or risks of hardware failure/damage in the field can be known and understood in advance, and proper measures can be taken to mitigate those issues if they occur in the field. Overall, HIL evaluation is a highly recommended approach, and it significantly increases the confidence level that the communication system works correctly and consistently, the desired control and operation performance of DERMS is achievable, and the distribution system is stable and regulated with integrated DERMS technology. The key challenge of HIL evaluation of DERMS technology is to set up the testbed and fully replicate the field deployment with a real-world distribution system model, hardware controllers, hardware inverters, and communications. 
A generic hardware testbed for evaluating coordinated control among advanced distribution management systems (ADMS), other utility management systems (e.g., DERMS and microgrid controller), DERs, and legacy utility equipment controllers (e.g., LTC, capacitor banks and voltage regulator controllers) was established in [16] to provide a realistic and trustworthy laboratory testing environment to evaluate the performance and functionalities of ADMS system. An advanced HIL platform was established at NREL's Energy System Integration Facility (ESIF) leverging the HIL capability in [16] to evaluate the control performance of voltage regulation of DERMS with real-time simulations of a real-world utility distribution system with a high penetration of photovoltaics (PV), an integrated real hardware controller (ADMS and DERMS), and power hardware (DERs) [17]. Yet, HIL testing of DERMS is still an underdeveloped area that requires more research and engineering effort to move the DERMS technology forward; therefore, this paper expands on [17] and goes beyond existing work to develop a generic HIL simulation framework by focusing on evaluating DERMS controller(s) and it provides helpful insights into developing the integrated platform and evaluating the performance of DERMS. One example is given to apply the generic framework and demonstrate the benefits of the advanced HIL platform, e.g., helping utility partners understand the advantages of using DERMS to manage large-scale DER integration and to mitigate the risks of technology integration. In particular, we focus on demonstrating how to replicate field deployment, and how to test communication and functionalities of the DERMS.

The major contributions of this paper are summarized as follows: this paper (1) proposes an generic HIL framework for DERMS performance evaluation and uses one real-world project as example to apply for the generic framework; (2) presents the detailed information and implementation process of each element for the example HIL setup, and especially provides guidance on testing and tuning the coordinated system in terms of communications and algorithm convergence; (3) evaluates the impacts of different execution time steps of the DERMS coordinator and local controllers on the system voltage regulation performance, identifies the time step of DERMS coordinator affect system performance significantly, and suggests a fast operating DERMS coordinator is key for satisfactory control performance and (4) compares the HIL evaluation results with baseline scenario (volt-VAR smart inverter) to demonstrate the performance and efficacy of the DERMS coordinated control, and verifies the feasibility of the HIL platform for DERMS performance evaluation.

\section{Integrated Framework of the HIL Setup}

To have a generic HIL setup that can test various utility distribution systems, vendors' ADMS, and DERMS, the integrated HIL framework needs to have the following features and capabilities:

1. Real-time simulation of a large distribution system that can accurately simulate and represent the steady-state and transient dynamics of the utility distribution systems for the study of system-wide coordination. Further, the simulated DERs should be able to emulate the real response and dynamics of the physical DER inverters and the interactions with the distribution grid.

2. The ADMS should be configured based on the database provided by the utility partner, and it can be modified to accommodate the needs of the HIL testing and evaluation.

3. The DERMS should communicate with upstream ADMS and downstream DER inverters. Based on the request from the ADMS, the corresponding control and operation algorithm should be enabled to dispatch the DERs. If the source code of the DERMS algorithms cannot be accessed, black-box testing should be possible.

4. Hardware DER inverters should receive the dispatched setpoints and commands from the DERMS and perform the expected operation to output the active and reactive power.

5. Communication and data exchange among elements should use standard industry communications protocols so that they can be deployed as they would be in a utility environment. 
Figure 1 shows the generic HIL setup that incorporates all the features into an integrated framework. This integrated setup comprises the HIL platform (real-time simulation platform and power amplifiers, etc.) common for all projects and systems under test (ADMS, DERMS, and hardware DER inverters) for a specific project.

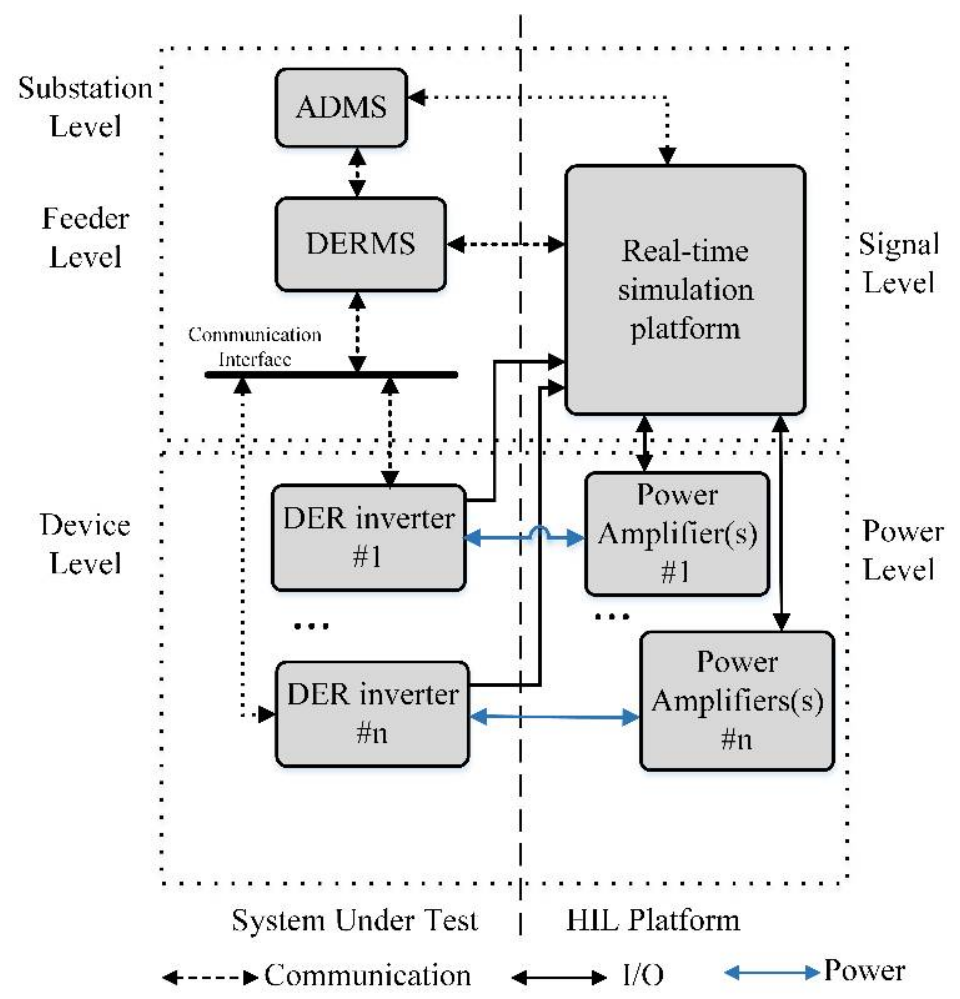

Figure 1. Generic schematic diagram of the HIL platform for testing the distribution automation system.

The real-time simulation platform can simulate the distribution system in real-time, and it can provide analog inputs and outputs for power-hardware-in-the-loop (PHIL) and communications channels for the controller under test. Power amplifiers include a grid simulator and a DER emulator (if no physical PV panels/battery are installed). A grid simulator is a high-power, high-bandwidth power electronics amplifier to reconstruct the simulated voltage from real-time simulation to physical voltage and enforce the real power virtually exchanged between the real-time simulator and the actual hardware under test (HUT). A DER emulator is also a power electronics amplifier that can emulate the physical dynamics of the DC side of a DER inverter. The system under test includes elements from the device-level DER inverters, to the feeder-level DERMS, to the substation-level ADMS. The communications network and link are developed to allow them to communicate with each other and to interact with the distribution power system model in real-time. As shown in Figure 1, the integrated framework integrates the signal-level and power-level HIL, which is commonly used to test a specific component as well as the complete distribution system with hardware controllers and devices. Note that the communications interface is optional depending on whether the DERMS downstream and the hardware inverters are assigned in the same network and communicate to each other using the same protocol.

\section{Development of the HIL Platform for HCE Project}

This section presents the realization of the integrated HIL platform to demonstrate the feasibility of the generic HIL framework. We use the HIL platform developed at the National Renewable Energy Laboratory to evaluate the grid automation system for a local utility in Colorado, Holy Cross Energy (HCE). HCE has the goal to achieve 70\% clean energy by 2030. Thus, pilot projects for deploying renewable energy and related technology are developed in the field. A field demonstration with the same grid automation system 
was planned after the laboratory testing which validates functionality and communication of the grid automation system, proves the concept, and de-risk technology integration in the field.

The platform configuration, shown in Figure 2, includes the co-simulation (OpenDSS, testbed coordinator, and OPAL-RT) of the HCE distribution feeder, a three-phase grid simulator with independently controlled phases, and a PV emulator with multiple modules and the system under test includes the substation-level controller Survalent ADMS, feederlevel controller DERMS coordinator, two DERMS DER local controllers, and six DER hardware inverters. This example platform accurately represents the dynamics of the real-world distribution system and evaluates real controllers and power hardware devices with standard communication protocols, thus providing a realistic laboratory testing environment. The following sections explain each main element in detail along with the communications protocols and data exchange among them.

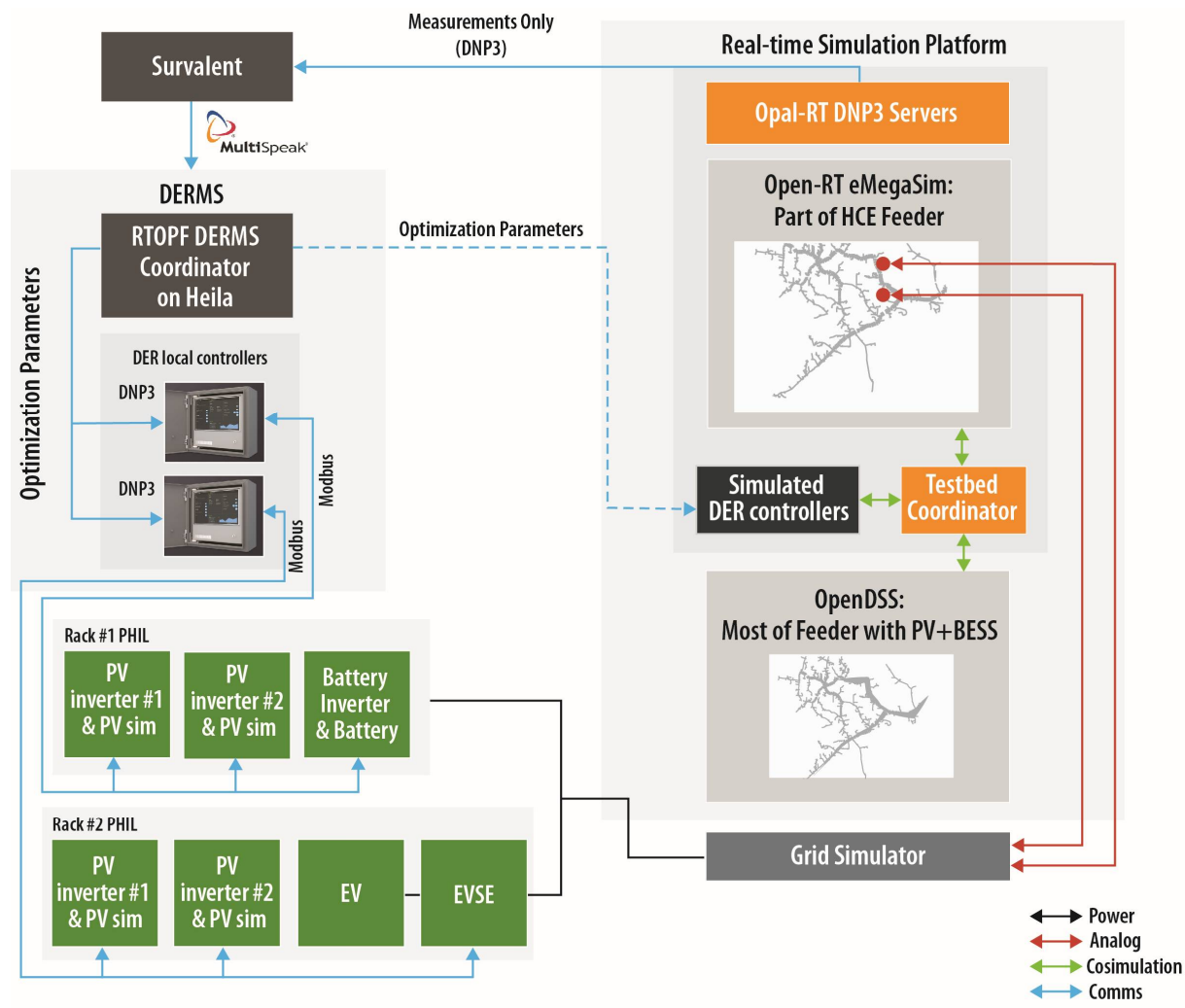

Figure 2. Schematic diagram of the HIL platform of the example project.

\subsection{Co-Simulation}

An actual distribution feeder located in Colorado in the service territory of the utility HCE is shown in Figure 3. The green line is three-phase or polyphase, the yellow line is Phase A, the red line is Phase B, and the blue line is Phase C. The peak load of the feeder is $11 \mathrm{MW}$. With nearly 4000 three-phase nodes, the feeder is large compared to many standard test systems. The renewable penetration level in the feeder is approximately $15.6 \%$ of peak load, including PV, battery, and electric vehicle (EV). To simulate the full model of this large system and accurately represent the dynamics in real-time, co-simulation is used that includes quasi-static time-series simulation in OpenDSS and an electromagnetic transient (EMT) real-time simulation in OPAL-RT. The simulation time step resolutions in OpenDSS and OPAL-RT are $2 \mathrm{~s}$ and $100 \mu \mathrm{s}$, respectively. 


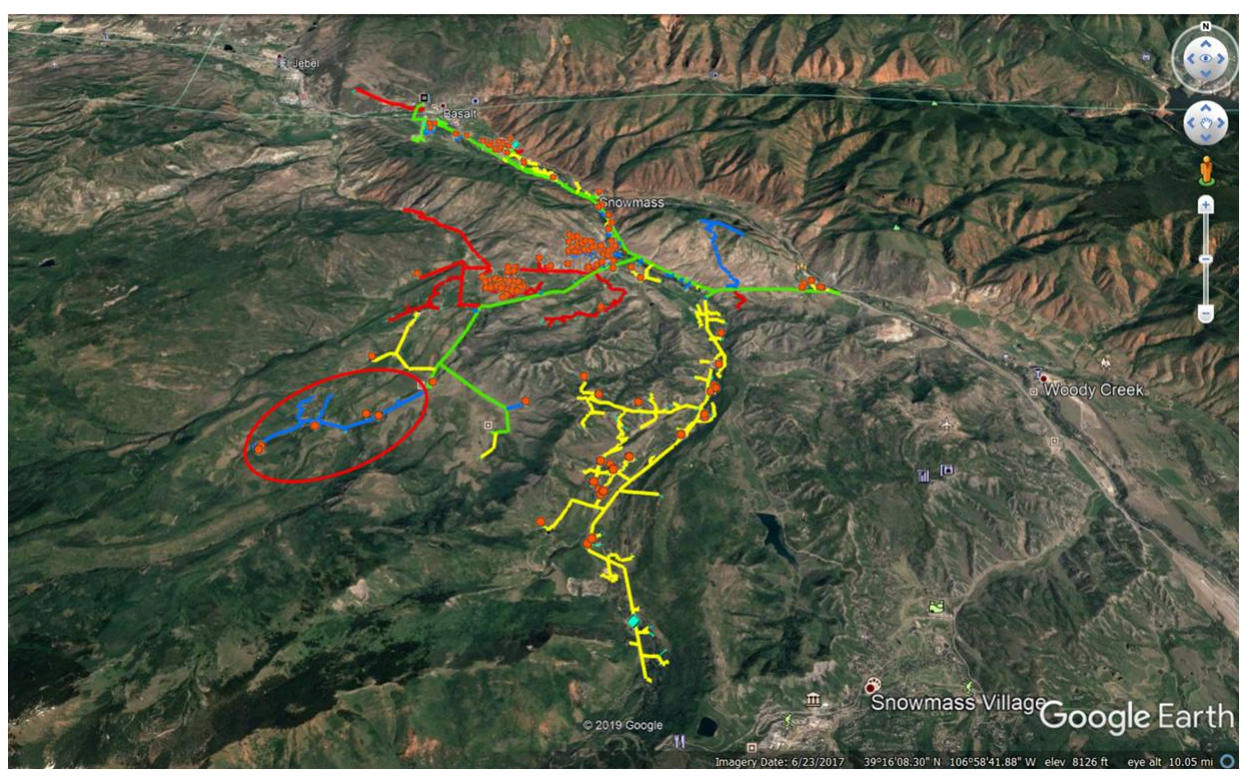

Figure 3. Topology of the real-world utility distribution feeder shown in Google Earth. Provided by HCE.

The topology of the utility distribution feeder is shown in Figure 3. The feeder consists of 163 all-electric homes-i.e., the household equipment includes residential rooftop PV, residential battery energy storage system, heating, ventilating, and air-conditioning unit, and electric water heaters. The locations of the all-electric homes are highlighted by the red dots in Figure 3. A large portion of the feeder is modeled in OpenDSS. The load and PV profiles are generated using the AMI data recorded in the field for the feeder modeling as detailed in [18].

The feeder section encircled by the oval in Figure 3, referred to as subtree, is modeled in OPAL-RT using the EMT simulator eMEGASIM. This subtree having 72 nodes is selected because it represents the typical circuit structure of HCE and it has the DERs required for the PHIL evaluation. The subtree head is modeled as a Thevenin circuit with Zs and Vs as the source impedance and voltage, respectively. Zs is the short-circuit impedance at the bus where the subtree is connected to the main feeder and it is obtained from the offline feeder fault study. The magnitude and angle of Vs come from OpenDSS power flow at each time step in the real-time co-simulation. In addition to running the power flow, the OPAL-RT also interfaces with the power hardware via analog inputs and outputs and communicates the selected measurement data from the power flow to the ADMS using industry-standard Distributed Network Protocol 3 (DNP3).

A co-simulation manager developed in Python for the ADMS test bed [16], referred to as testbed coordinator, is used to perform the co-simulations in this work. The testbed coordinator uses the Hierarchical Engine for Large-scale Infrastructure Co-Simulation (HELICS) [19] framework and ensures the synchronous data exchange between OpenDSS and OPAL-RT in the real-time co-simulation through time. As shown in Figure 2, the testbed coordinator combines OpenDSS, OPAL-RT, load profiles, load models, and simulated DER controllers to enable data exchange among entities with a programmable timing mechanism. The inputs to the DERMS are the power flow measurements from the feeder modeled in OpenDSS and OPAL-RT. At each time step, the measurements from OpenDSS are collected first and sent to OPAL-RT. The OPAL-RT combines these measurements with those from the subtree and sends to the ADMS. The ADMS sends them to the DERMS coordinator. This process is repeated at every time step of the co-simulation. 


\subsection{Controller Hardware-in-the-Loop (CHIL)}

In this example HIL platform, the controller under test includes the Survalent ADMS, the Heila DERMS coordinator, and two Heila local controllers. They are all commercially available grid automation products. To emulate the field deployment, the ADMS receives real-time measurements (the same variables from the AMI data) from the co-simulation through the DNP3 protocol. The data are then passed to the DERMS coordinator as inputs for the optimization algorithms. Further, the ADMS works as the brain of the distribution automation system to control the legacy devices and coordinate with the DERMS.

Therefore, the Survalent ADMS performs two key functions: (1) Enable/disable the DERMS algorithm to provide the grid services to the distribution feeder and set voltage limits (for voltage regulation) and power references (for VPP control) and (2) to emulate a supervisory control and data acquisition (SCADA) system for data visualization and a gateway to communicate the measurements to the Heila DERMS coordinator.

The NREL team developed the DERMS algorithms based on the real-time optimal power flow (RT-OPF) algorithm from [20]. The high-level schematics of the DERMS optimization algorithm are shown in Figure 4a. The measurement variables are the voltages at selected nodes and the active and reactive power at the feeder head which are sent to the RT-OPF coordinator to run the optimization algorithm. The RT-OPF coordinator also receives the control settings and operation constraints from upper streams, such as distribution system operator (DSO) through DMS/ADMS. The outputs of the coordinator are the dual variables (gradients) for each DER distributed/local controller. Each DER local controller receives the dual variables from the coordinator and local measurements from the DERs (e.g., battery state of charge) and then outputs the optimal power setpoints for each DER. Coordinatively, the DERMS achieves the system-level goal of enforcing limits (voltage regulation) and the device-level goal of minimizing customer bills in this project. $\lambda \_i, \mu \_i, \gamma \_i$ are dual variables that capture the system over-voltage, under voltage, feeder head power tracking over the power injection at node $i$. Note that each dual variable includes two variables, the first one corresponding for active power and the second one corresponding for reactive power. $P_{P V, t}^{k}, Q_{P V, t}^{k}$ and $P_{B E S S, t}^{j}, Q_{B E S S, t}^{j}$ are realtime power output from PV connected at node $i$ and output from Battery connected at node j. $P_{P V, t+1}^{*}, Q_{P V, t+1}^{*}, P_{B E S S, t+1}^{*}, Q_{B E S S, t+1}^{*}$ are optimal power setpoints for devices. DERs parameters include inverter capacity, SOC limit, charging/discharging power limit, which can be hardcoded in the local controllers.

Another important aspect of deploying RT-OPF DERMS is to determine the communication architecture. Therefore, Figure $4 \mathrm{~b}$ presents the proposed communication architecture to deploy this RT-OPF DERMS. The measurements for RT-OPF coordinator can be collected through AMI and SCADA through DNP3 protocols, and the resolution is seconds to minutes. The signals from DSO can be sent through enterprise-level communication protocols, such as CIM, ICCP and MultiSpeak, and the resolution can be minutes to hours. The outputs of the coordinator are sent to the local controllers through DNP3/UDP with a resolution of seconds to $1 \mathrm{~min}$. For the local controller, it receives local measurements and sends the setpoints to DERs through ModBus communication protocols with a resolution of milliseconds to $20 \mathrm{~s}$. Note that the local controllers can also communicate with the DERs through other protocols, such as OpenADR, IEEE 2030.5 and SunSpec ModBus. 


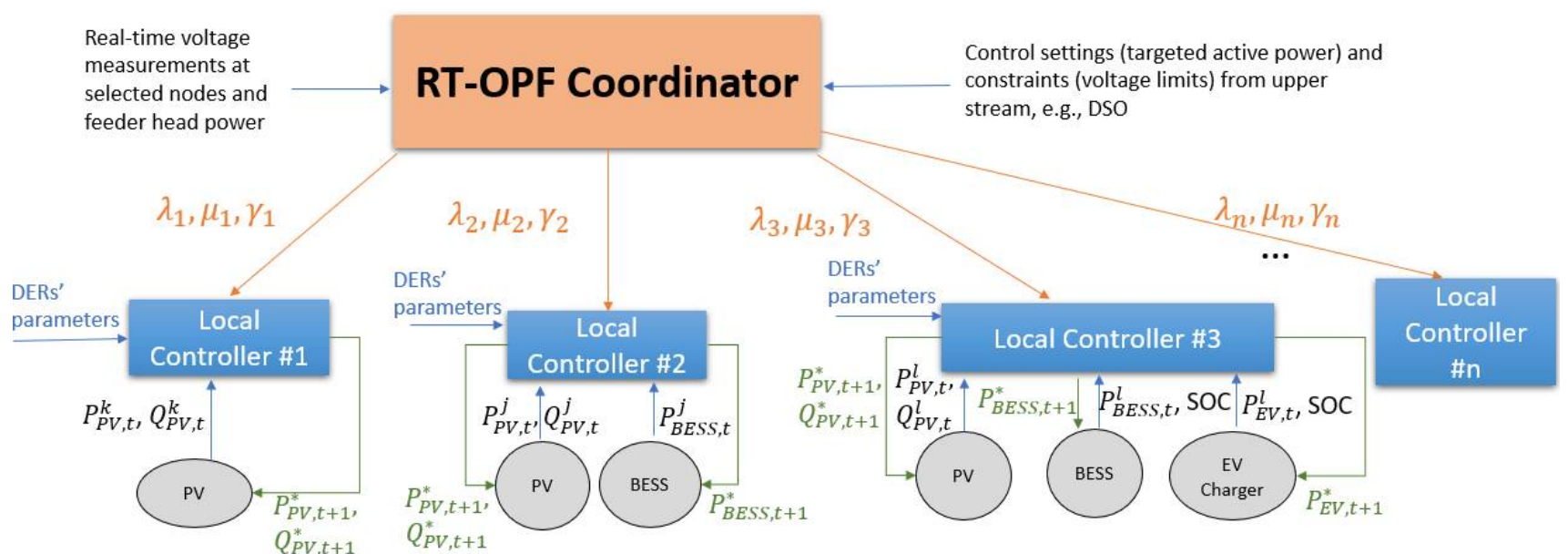

(a)

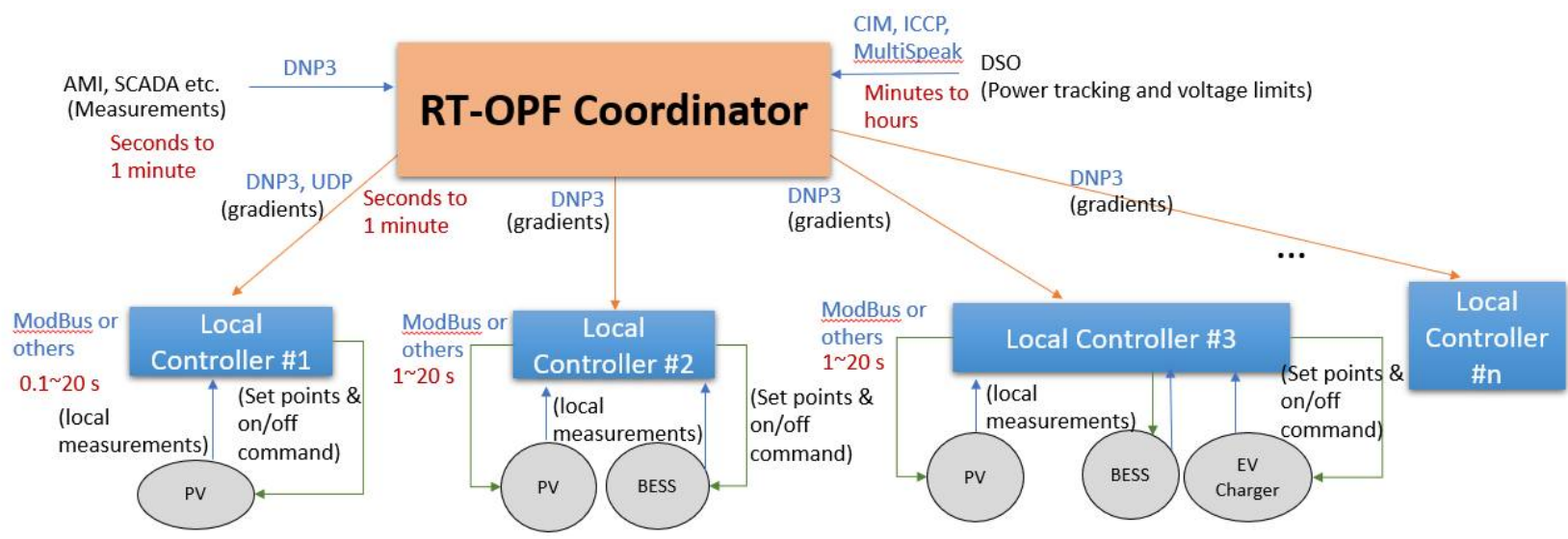

(b)

Figure 4. RT-OPF: (a) high-level schematics of the DERMS optimization algorithms and (b) communication architecture of implementation.

The DERMS coordinator algorithm is embedded in a commercial Heila EDGE controller for this project which is referred to as the Heila DERMS coordinator. Further, two local DER controller algorithms are implemented in two additional Heila EDGE controllers, referred to as Heila local controllers. The remaining 161 local DER controllers associated with the all-electric homes are implemented in the computer running the co-simulations. The high-level implementation of the Heila coordinator is shown in Figure 5. The Heila coordinator receives the measurement inputs from the ADMS every $3 \mathrm{~s}$ through the MultiSpeak standard. The inputs include the vectors $P_{s p}$ for the feeder-head active power set points, $P_{p c c}$ for the feeder-head active power, $V_{\text {meas }}$ for the voltage measurements, $V_{\text {lim }}$ for the voltage limits. After receiving the data inputs, the Heila control functions execute standard commands for data handling, such as fetching data from ADMS, send start/stop commands, and set power dispatch commands. In each 3 s interval, the DERMS coordinator computes the optimization parameters, i.e., the voltage and power gradients based on the dual variables $\lambda, \gamma$, and $\mu$. The optimization parameters are sent to the downstream communications module and to the Heila local controllers every $1 \mathrm{~s}$ through DNP3. 


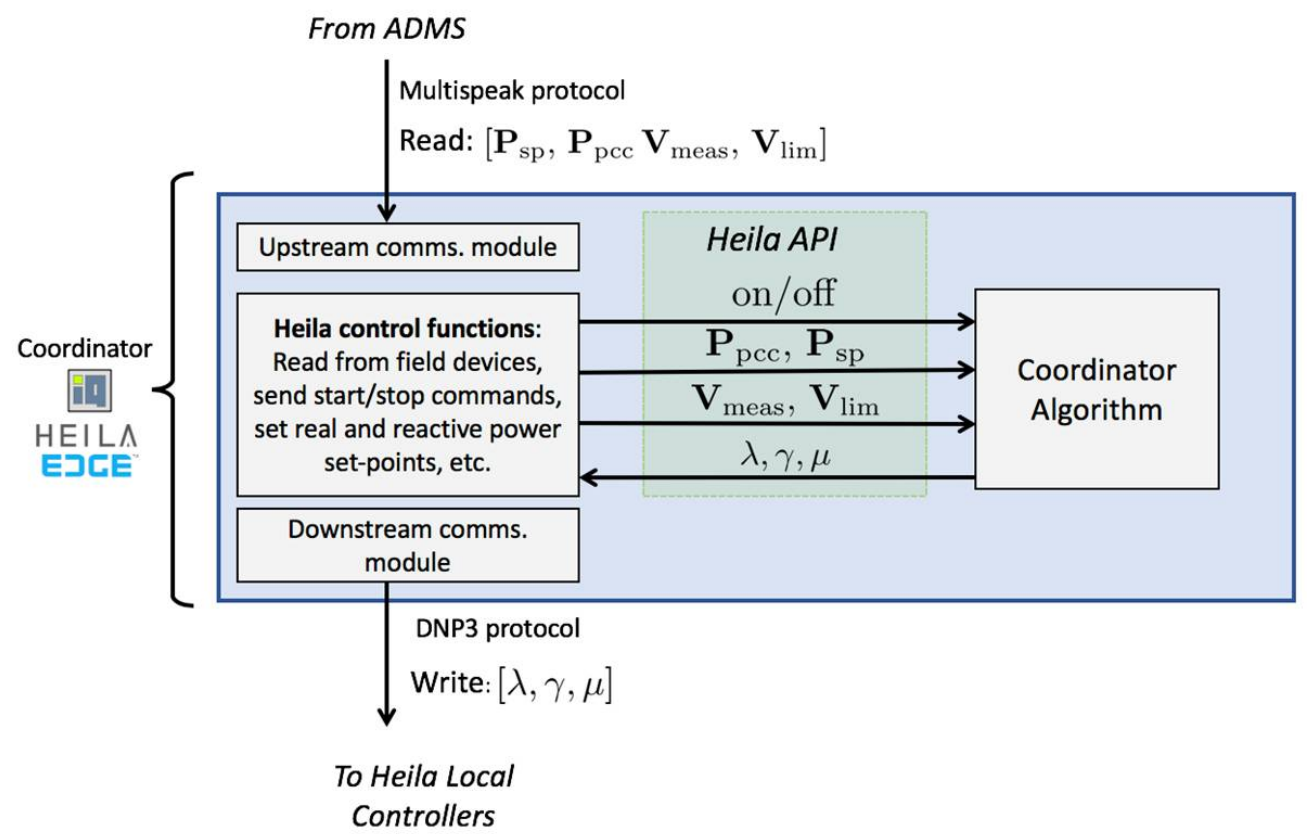

Figure 5. Illustration of Heila DERMS coordinator implementation.

The implementation and the data flow in the Heila local controller are shown in Figure 6. The Heila local controllers collect the coordinator outputs and the local DER measurements from the DER inverters and pass them to the local DER controller algorithm. The local DER measurements include active and reactive power statuses and the state of charge of the battery energy storage systems. Based on these inputs, the local DER controller algorithm computes the optimal power setpoints for the physical DER inverters in the PHIL. The Heila controllers send the setpoints to the physical DER inverters at every $2 \mathrm{~s}$ through Modbus.

From Coordinator

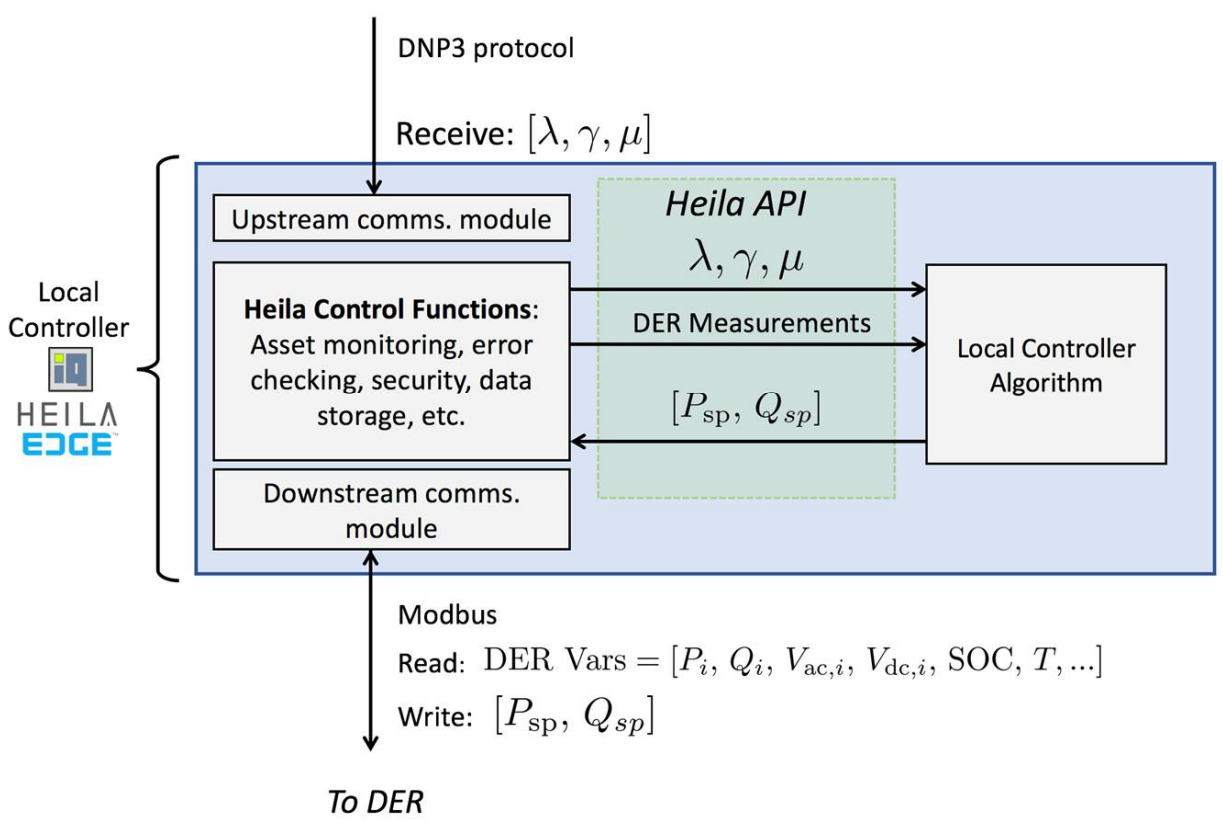

Figure 6. Illustration of the Heila local controller implementation. 
Because of space and budget constraints, most DER distributed controllers are validated via a simulation environment. To emulate field deployment and provide realistic testing results, all simulated DER local controllers are implemented to emulate the hardware DER Heila local controllers: (1) program in the same language; (2) receive the dual variables from the Heila coordinator with the same rate; (3) receive the local measurements from DERs with the same rate, and (4) have the same execution time to run the algorithm and output the set points. Testing the simulated DER local controllers is called controller-software-in-the-loop (CSIL). CSIL testing provides an excellent complement to traditional controller-hardware-in-the-loop (CHIL) simulation with fast-code modification and code debugging, trustable results, and performance evaluation of control source code [21]. The simulated DER local controllers receive the optimization parameters from the DERMS coordinator via an HTTP interface and receive the power flow measurements from OpenDSS through the testbed coordinator. Based on these inputs, they compute the optimal power set points and send them to the simulated DERs in OpenDSS. They run every $2 \mathrm{~s}$ as the Heila hardware controller, and the inputs and outputs are synchronized with the co-simulation through the testbed coordinator. This generates real-time responses of simulated local controllers as if they were running in real hardware controllers, such as the Heila local controllers. The mixed CHIL and CSIL essentially creates a real-time and realistic testing environment and results for distributed DERMS deployed in real life.

\subsection{Power Hardware-in-the-Loop (PHIL)}

The DER inverter power hardware is integrated using PHIL to output the desired amount of power based on the power setpoints from the Heila local controllers; therefore, each DER inverter is configured within the same subnetwork as the Heila local controller and to be controlled by an external controller via Modbus Transmission Control Protocol (TCP).

The DER inverters are connected to a grid simulator that reconstructs the simulated voltages where the DERs are connected in OPAL-RT. The measured currents and voltages of the DER inverters are fed back to OPAL-RT to control the current source; thus, the use of a measurement-control feedback loop replicates the actual dynamics and response of the DER inverters. There are six DER inverter HUT as shown in Figure 2 that are installed in two DER racks. Each rack represents a point of common coupling (PCC) of an actual all-electric home in the field. Table 1 presents the high-level descriptions of these racks.

Table 1. List of DERs.

\begin{tabular}{ll}
\hline \multicolumn{1}{c}{ Rack Components } & \multicolumn{1}{c}{ Description } \\
\hline Rack 1: & $\begin{array}{l}\text { Both PV inverters produce active and reactive power, } \\
\text { and the battery inverter produces only active power. }\end{array}$ \\
- $\quad$ 3-kW SMA PV inverter & The PCC voltage and current are fed back to OPAL-RT. \\
- $\quad$ 5-kW Fronius PV inverter & $\begin{array}{l}\text { The DC side of the PV inverters is powered by PV } \\
\text { emulators, and the battery inverter is powered by a } \\
\text { - } \quad \text { 5-kW SolarEdge battery inverter battery. }\end{array}$ \\
\hline Rack 2: & Rack 2 has the same PV units as Rack 1. The EV is \\
- $\quad$ 3-kW SMA PV inverter & connected through an EV supply equipment and can \\
- $5-\mathrm{kW}$ Fronius PV inverter & $\begin{array}{l}\text { only draw active power from 0 to rated power, i.e., it } \\
\text { cannot discharge or provide reactive power. }\end{array}$ \\
\hline
\end{tabular}

A grid simulator (RS90 manufactured by California Instruments) is used to interface both the DER racks with the subtree simulated in OPAL-RT. The grid simulator is a controllable AC power source and is used to reconstruct the nominal voltages corresponding to the simulated voltages in OPAL-RT at the DER locations. Racks 1 and 2 are connected to phases A and B, respectively using single-phase isolation transformers each rated for $15 \mathrm{kVA}$ for protection. 


\section{Implementation and Testing of the HIL Platform}

\subsection{Develop Communications}

The HIL platform includes multiple communications paths that use standard industry communication protocols, which are listed in Table 2. The Internet Protocol (IP) network is assigned first based on the layers shown in Figure 1. The device/controller in the same layer has the same subnet, which reflects the realistic implementation in the field because different layers are geographically far from each other, and different subnets are usually used for communications. Each Heila coordinator and local controller has two IP addresses: one for upstream communications and another one for downstream communications. The IP addresses for the downstream communications in the Heila local controllers are assigned the same subnet as the DER inverters. The ADMS and the Heila coordinators have different subnets. Therefore, an internet exception is implemented in the ADMS to enable communications between them.

Table 2. Communications in HIL platform.

\begin{tabular}{ccc}
\hline Communications Path & $\begin{array}{c}\text { Communications } \\
\text { Protocol }\end{array}$ & $\begin{array}{c}\text { Data Publish/ } \\
\text { Subscribe Interval }\end{array}$ \\
\hline OPAL-RT and ADMS & DNP3 & $100 \mu \mathrm{s}$ and $1 \mathrm{~s}$ \\
ADMS and DERMS coordinator & MultiSpeak & $1 \mathrm{~s}$ and $1 \mathrm{~s}$ \\
Coordinator and Heila local controller & DNP3 & $3 \mathrm{~s}$ and $2 \mathrm{~s}$ \\
Local controller and DER inverter & Modbus & $2 \mathrm{~s}$ and $2 \mathrm{~s}$ \\
\hline
\end{tabular}

Then, each device is configured to ensure that the correct settings (IP address, TCP port, point number, communications protocols, master/slave relationship, and link address) are mapped. Next, the data publish/subscribe time intervals in each element need to be defined. Except for the ADMS, all the elements use an execution time step as the interval. Note that the publish/subscribe intervals in the ADMS are determined by multiple trial tests that enable step changes in OPAL-RT and change the publish/subscribe intervals in the ADMS to see how long it takes for the Heila coordinator to receive the step changes from OPAL-RT. One second is the smallest time interval that includes the inherent time delays and latencies in the two paths (between OPAL-RT and the ADMS and between the ADMS and the Heila coordinator). Table 2 lists the data publish/subscribe intervals.

Extensive communications testing is performed before deploying each element in the whole platform. Specifically, we ensured that the communications between OPAL-RT and ADMS, ADMS and Heila coordinator, Heila coordinator and local controllers, and Heila local controllers and DER inverters were working correctly before proceeding to the co-simulation.

\subsection{Implement Co-Simulation}

The majority of the HCE feeder is simulated in OpenDSS, and a subtree is simulated in OPAL-RT. To set up the co-simulation, a User Datagram Protocol (UDP) communications block is developed in both simulation platforms, and the settings-such as IP address and port number-are then defined. At each time step in the co-simulation, the OpenDSS sends the magnitude and angle of the subtree head voltage to OPAL-RT and the OPAL-RT sends back the active and reactive powers to close the loop. The subtree head voltage information received from OpenDSS is used to set up the voltage source of the subtree in OPAL-RT. Additional variables including current magnitude, active and reactive powers are also exchanged between OpenDSS and OPAL-RT to compare the power flow. Further, a set of 167 variables needed for the DERMS algorithm including the 161 voltage measurements and the feeder head active and reactive powers are sent from OpenDSS to OPAL-RT. 


\subsection{Implement $A D M S$}

A remote terminal unit (RTU) is developed in the ADMS to include all the measurement data from OPAL-RT, and a communications line is established to link all the data with specified communications settings. The ADMS in the field polls the measurements from the meters at a fixed time interval using DNP3. To emulate this, we set up the ADMS to collect the measurements from OPAL-RT using DNP3 every $1 \mathrm{~s}$ which are then sent to the Heila coordinator using MultiSpeak standard (Version 5.0), and the interface type is 'Outage Analysis' with the method of 'GetLatestSCADAAnalog'. The setpoints (voltage upper and lower limits and feeder-head active power references) and enabling signal (voltage regulation) are implemented as "Set-Point" in the RTU to change them in real-time, and they are sent to the Heila coordinator together with the measurements.

\subsection{Implement DERMS}

The RT-OPF DERMS algorithms were implemented and tested through pure simulation first to ensure that the algorithms were implemented correctly and that the performance (voltage regulation) was achieved. Then the Python codes of the coordinator and local controller are uploaded and integrated into the hardware controllers. Extra tuning effort is needed because the various delays related to the hardware implementation (e.g., multiple communications paths, data encoding and decoding in the hardware, and communications request and response) all affect the performance of DERMS.

Essentially, the performance of the RT-OPF DERMS is a function of hyperparameters, mainly including global gradient step size in the coordinator, local step size, and the weights of the active power curtailment and reactive power generation in the DER local controllers. Because the gradient step size in the coordinator dominates the convergence and performance of the RT-OPF, the first step is to tune this parameter. Increasing its value accelerates convergence and could result in oscillations and instability, and reducing its value decelerates convergence and could achieve poor performance. The recommended value of gradient step size in the coordinator can be found in [18], and it can be slightly increased/decreased with the step of $10^{-4}$ based on the performance.

The second step is to tune the parameters in the local controllers. The gradient step size in the local controllers is tuned to achieve a "reasonable" response time (a settling time of 1-2 min). Also, the weight of the active power term in the cost function is selected relatively higher in comparison to the reactive power term so that the voltage regulation is accomplished primarily by the reactive power compensation without relying heavily on the active power curtailment.

After setting the parameters in the local controllers, the gradient step size in the coordinator is adjusted again to ensure convergence because the standard learning rate parameter needs multiple iterations of adjustment in the gradient descent algorithm. The convergence of the RT-OPF is achieved through tuning those parameters via multiple iterations, and the optimum parameters were found for the convergence and the best performance.

\subsection{PHIL Configuration and Implementation}

A schematic diagram of the PHIL implementation for each rack is presented in Figure 7, which shows the interface algorithm in OPAL-RT and the signal flow between the hardware and the real-time simulation.

The DER inverters HUT at each PCC is modeled as a current source connected to the subtree of the distribution feeder. The RMS value of the DER inverter terminal voltage, $v$, is calculated to re-create a sinusoidal wave for the grid simulator with a fixed frequency and phase angle $\left(60 \mathrm{~Hz}\right.$ and $\left.0^{\circ}\right)$. This is different from traditional PHIL testing, which uses the scaled sinusoidal wave of $v$ directly for the grid simulator. Thus, the grid simulator replicates the full dynamics of the simulated voltage, $v$, for the DER inverters. Re-creating the sinusoidal wave with RMS of $v$ and fixed frequency and angle, however, can produce a relatively clean waveform and still represent the system phasor dynamics. This is acceptable because the focus is to evaluate how the DERMS collectively respond to the 
request from the ADMS and dispatch the DER inverters and how DER inverters respond to the dispatch signals from the DERMS at the feeder-level rather than the DER inverters' device-level dynamics and stability.

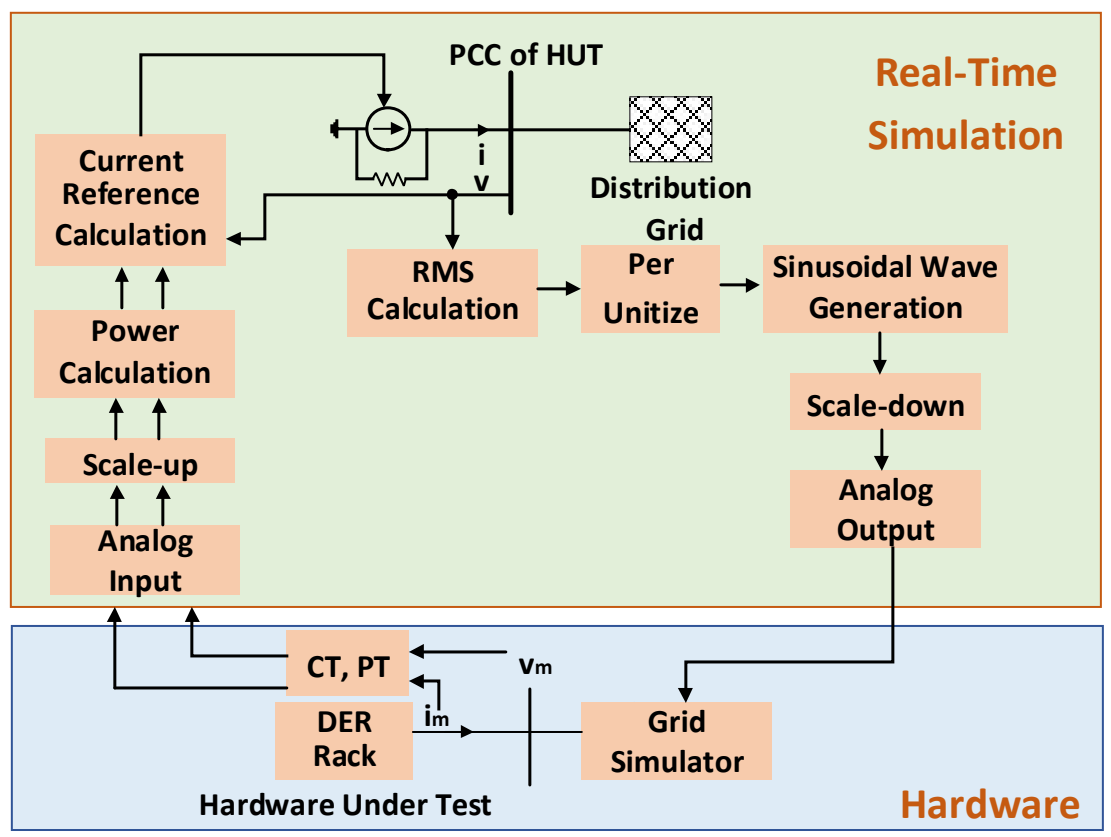

Figure 7. The PHIL interface algorithm implemented in OPAL-RT.

The actual current and voltage of the DER racks are measured through a current transformer and potential transformer, respectively, and sent back to OPAL-RT through analog inputs. The current and voltage are then scaled up to actual values in the DER racks, and then the actual power is calculated. Noticeable artificial reactive power bias (approximately 500 volt-ampere reactive [VAR]) is observed in each rack because of the different delays in the current sensor and voltage sensor. Additional compensation is added to cancel out the bias in the reactive power [22]. The simulated voltage, $v$, is used to generate the synchronized current reference to control the current source and close the PHIL loop.

Sample PHIL testing results are shown in Table 3. Note that the power references ( $P^{*}$ and $\mathrm{Q}^{*}$ ) are sent from the Heila local controllers with manually set values. The last two rows are the power feedback to OPAL-RT. The calculated tracking errors for the active and reactive power are less than $1 \%$ and $3 \%$, respectively; thus, this PHIL interface algorithm ensures the stability of connecting multiple HUT at one PCC and multiple PHIL PCCs into the system, and it guarantees the accuracy of replicating the actual output power and dynamics from physical DER hardware inverters.

Table 3. Sample PHIL Test Results.

\begin{tabular}{ccccccc}
\hline & \multicolumn{3}{c}{ Rack 1 } & & \multicolumn{3}{c}{ Rack 2 } \\
\cline { 2 - 6 } & $\begin{array}{c}\text { SMA } \\
\text { Inverter }\end{array}$ & $\begin{array}{c}\text { Fronius } \\
\text { Inverter }\end{array}$ & $\begin{array}{c}\text { LG } \\
\text { Battery }\end{array}$ & $\begin{array}{c}\text { SMA } \\
\text { Inverter }\end{array}$ & $\begin{array}{c}\text { Fronius } \\
\text { Inverter }\end{array}$ & EV \\
\hline $\mathrm{P}^{*}$ & 1500 & 2000 & 1300 & 2000 & 2500 & $20 \%$ \\
$\mathrm{P}_{\mathrm{m}}$ & 1525 & 1992 & 1333 & 2048 & 2490 & 1325 \\
$\mathrm{Q}^{*}$ & 500 & 1000 & 0 & 0 & 2000 & - \\
$\mathrm{Q}_{\mathrm{m}}$ & 529 & 1048 & 10 & 13 & 2033 & - \\
$\mathrm{P}^{*} / \mathrm{P}_{\mathrm{m}}$ & & $4800 / 4825$ & & & $5820 / 5846$ & \\
$\mathrm{Q}^{*} / \mathrm{Q}_{\mathrm{m}}$ & & $1500 / 1543$ & & & $2000 / 2056$ & \\
\hline
\end{tabular}




\section{Integration Test}

After establishing communications and implementing each element, all elements except the hardware inverters are connected for the stability test. Note that the two hardware Heila local controllers use the set points from the last step as the feedback measurements for the test. The operation procedures of evaluating voltage regulation performance are summarized as follows: run the co-simulation first, check if the ADMS receives the DNP3 data points, confirm if the DERMS coordinator receives the measurement data and setpoints, verify if the local controllers receive reasonable dual variables, enable the voltage regulation in the ADMS, view the dual variables and PV outputs from the dashboard of the selected local controllers, and observe if the system is stable and voltages are regulated within the operation limits from the ADMS data visualization system. If this stability test is successful, the hardware inverters are then connected for the integration function test.

\subsection{Function Test}

After the stability test, all the elements are integrated to test the communications and functionality. Each element has its own execution time step, and data exchange among elements is defined with a fixed interval; thus, tuning the time step and data exchange intervals is necessary to ensure that the DERMS optimization algorithms converge with the multi-time scale system. Note that the ADMS plays a key role in determining how fast the DERMS algorithms converge because it acts as a gateway twice to pass data between OpenDSS and the Heila DERMS coordinator. The general practice is to ensure that the ADMS is not slower than the execution time step of the receiver (e.g., coordinator). The integrated HIL platform with multiple timescales is illustrated in Figure 8, and the time step in each element is obtained through a tuning process to ensure that the DERMS optimization algorithms converge.

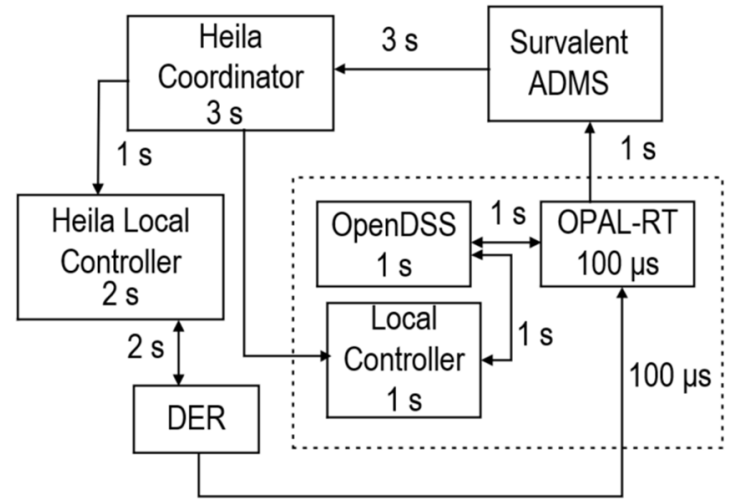

Figure 8. The integrated HIL simulation with multiple time steps.

To show the convergence of the DERMS algorithms, the preliminary test results of the voltage regulation are shown in Figure 9. The simulation starts from the baseline scenario without DERMS control, and then the DERMS is enabled by the ADMS near step 100. As shown from the results, the voltages converge near 140 steps, 40 steps after the control is enabled. Note that each time step is $1 \mathrm{~s}$, and the upper limit for voltage regulation is set to be 1.038 p.u. After the DERMS regulation, the voltages are regulated under the limit. These preliminary test results show that the DERMS works as expected to regulate the voltages within the limit and that the HIL platform can be used to evaluate the performance of DERMS. 


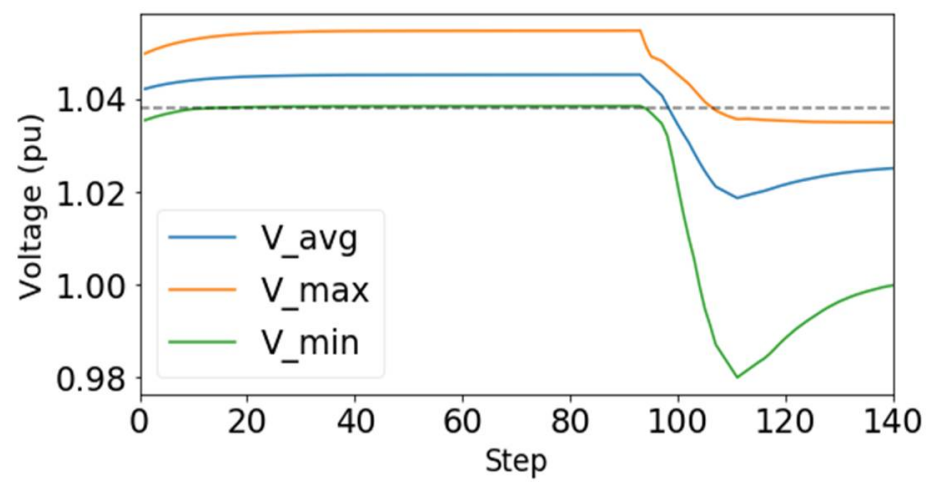

Figure 9. Preliminary functionality test results of voltage regulation.

\subsection{Time Step Test}

More work is carried out to examine the impact of the time steps in the DERMS on the control performance and to determine the time step resolution that leads to the optimal performance. We implemented various time steps in the DERMS coordinator and the local DER controllers.

Starting from the coordinator, $t=2,5,10,15$, and $20 \mathrm{~s}$, respectively, are used for the coordinator with a fixed time step $(2 \mathrm{~s})$ in all the distributed local controllers. The voltage regulation is enabled from the baseline scenario with high solar irradiance and overvoltage violations. Outputs of the coordinator for a selected local controller (overvoltage gradient $\lambda$ includes gradient 1 and 2), active and reactive power output of the corresponding PV inverter, and the PCC voltage measurements are recorded with different time steps in the coordinator. The results are shown in Figure 10a-c.
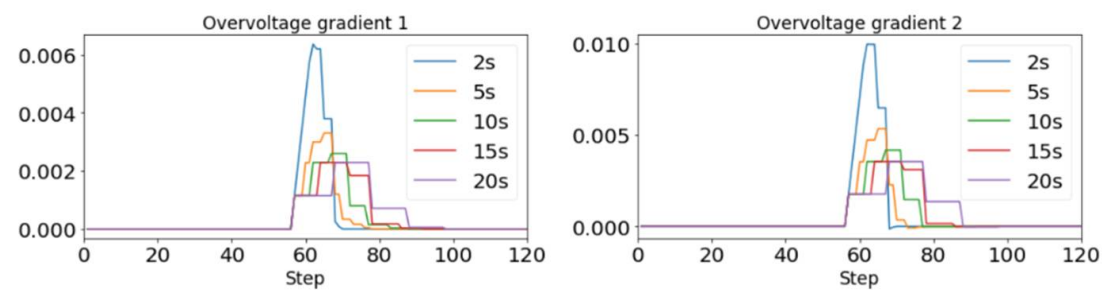

(a)
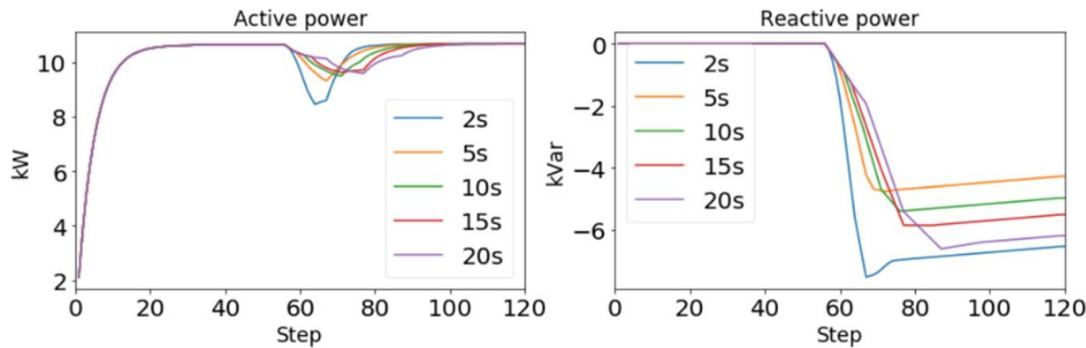

(b)

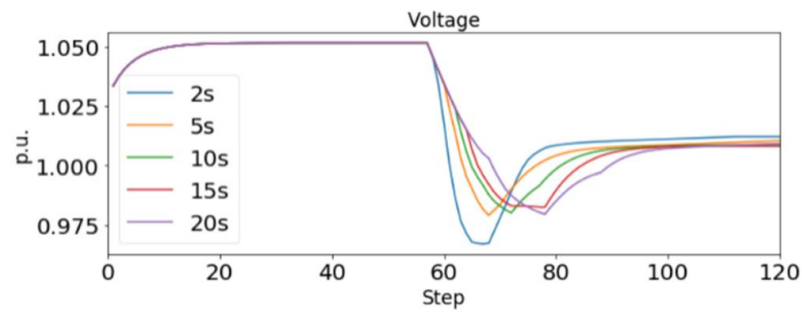

(c)

Figure 10. Impact on voltage regulation with different time steps in the coordinator: (a) outputs of the coordinator; (b) active and reactive power output of a selected PV inverter, and (c) voltage measurements. 
As shown in Figure 10a, the overvoltage gradient 1 and 2 have similar performance under the same time step of the coordinator, and both behave consistently under different time steps. The faster the coordinator runs (e.g., 2 s), the larger the overshoot and the faster the overvoltage gradients settle and reach their steady-state value. As the name suggests, the gradient is the slope rate of how fast/slow the local controller descends from the current location. The larger gradient values in the local controller drive the DER response faster and reach the target performance quicker. The active and reactive power outputs of the corresponding PV shown in Figure 10b indicate that outputs of PV with the smallest time step $(2 \mathrm{~s})$ reach steady-state the fastest, in less than 12 steps. The same response is observed in the PCC voltage of the corresponding PV shown in Figure 10c.

Similarly, we use $t=2,3,5,8$, and $10 \mathrm{~s}$ for all the local controllers with a fixed time step (10 s) in the coordinator, and we record the voltage regulation performance. The selected results are presented in Figure 11. As shown in Figure 11a, the overvoltage gradients 1 and 2 do not show the fastest response with the local controller running the fastest $(2 \mathrm{~s})$. A closer look at the settling times for each time step shows 97 steps with a 2-s time step, 92 steps with a 3 -s time step, 88 steps with a 5 -s time step, 91 steps with an 8 -s time step, and 87 steps with a 10-s time step. At the beginning of the test, the response of the system with a slower time step in the local controller is slower, and the voltage regulation performance is also slower. This means that the coordinator must respond to the overvoltage, and the output of the local controller running in the largest time step has the largest overshoot and gradient descent. The largest gradient descent, in turn, means that the local controller with the largest time step reaches a steady state faster (shown in Figure 10b, the response of the reactive power output). For the case with the smallest time step ( $2 \mathrm{~s})$, the response of the local controller is faster, and the voltage regulation performance is faster in the beginning. This results in the output of the coordinator running in the smallest time step having the smallest overshoot and gradient descent later, as shown in Figure 10a. The interactions between the coordinator and distributed local controllers indicate that the largest time step in the local controllers has the fastest response in voltage regulation and the smallest time step has the slowest response.

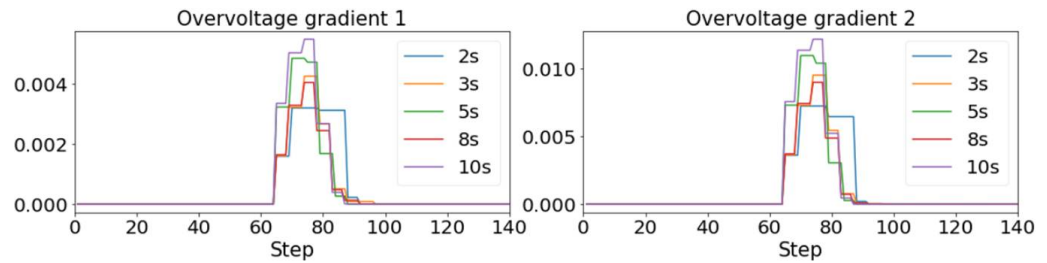

(a)
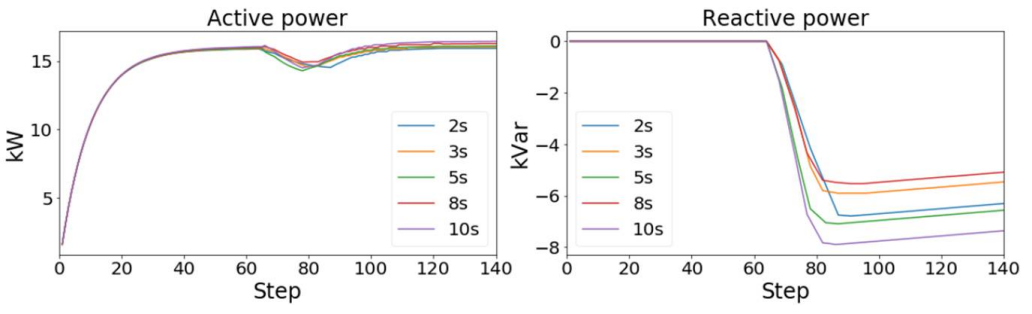

(b)

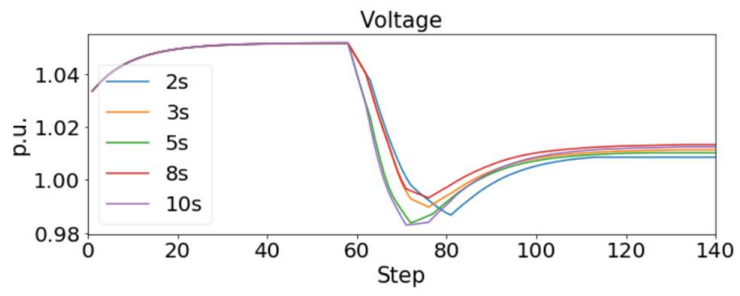

(c)

Figure 11. Impact on voltage regulation with different time steps in the local controllers: (a) outputs of the coordinator, (b) active and reactive power output of a selected PV inverter, and (c) voltage measurements. 
Comparing the two tests, we can conclude that the time step in the coordinator dominates the system response, and the time step in the local controllers is less influential. To obtain a satisfactory response, the key is to have the coordinator run in a smaller time step (e.g., $5 \mathrm{~s})$.

\section{Experimental Results}

In this section, the laboratory experiment results will be presented by using the developed advanced HIL platform to evaluate the voltage regulation performance of the coordinated system among the ADMS, DERMS and DERs. A 1-h (11:00 a.m.-12:00 p.m.) simulation window is selected, which is the peak time of solar irradiance, and a high-voltage issue is found. The load and PV profile data are derived from the AMI measurements collected in the field, but they are interpolated to 1-min resolution for simulating the near-realtime operation of the DERs. This HIL testing uses real feeder model data, commercial ADMS and DERMS controllers, physical hardware inverters, and standard communications protocols, which provide high-fidelity experimentation and trustable results, wherein actual hardware controllers and physical DER inverters interact with a real-time software model by leveraging CHIL and PHIL.

Because increasingly more DER inverters operate with smart inverter functions in volt/VAR mode, we simulate a baseline scenario with all PV operating with smart inverter functions following the volt/VAR curve for comparison.

\subsection{Baseline Scenario}

For the baseline scenario, all PV inverters operate in volt/VAR autonomous mode, and the battery inverters are disabled. The volt/VAR curve used in the simulation is shown in Figure 12, where the reactive power limits are set to $50 \%$ of the inverter active power rating. In this scenario, the distribution system model with the PV's autonomous control is simulated and implemented in OpenDSS, the power flow converges, and the system is numerically stable.

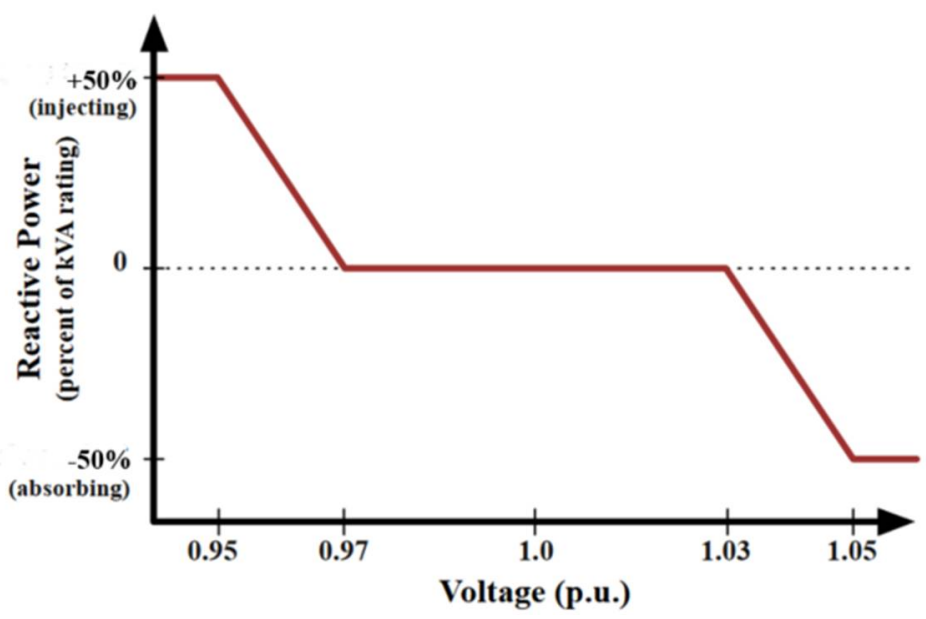

Figure 12. Volt/VAR curve used in the baseline simulation [23].

Figure 13 shows the results of the volt/VAR mode for the selected 1-h window (11:00 a.m.-12:00 p.m.), including all the control node voltage measurements. The results of the voltage measurements are very similar to those of the constant power factor mode in [17]. With the contribution of the reactive power in volt/VAR mode, the average system voltages are shifted down approximately 0.01 p.u. compared to the constant power factor mode; however, the voltages are still greater than the target operation limits of 1.038 p.u. This voltage limit is chosen instead of 1.05 p.u. to have a better comparison between baseline volt-VAR mode and control with DERMS. 


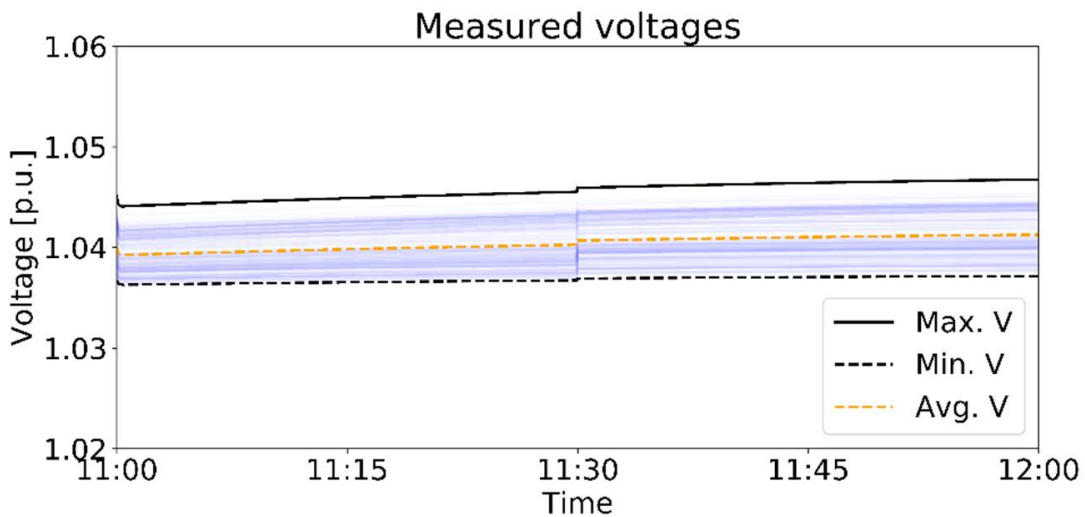

Figure 13. Results of volt/VAR control baseline scenario: average, maximal, and minimal voltage and all the voltages across the feeder.

\subsection{DERMS Control}

In this scenario, DERMS control is enabled in the ADMS server at the beginning of the test, and the upper and lower voltage limits for the DERMS voltage regulation are set as 0.96 p.u. and 1.038 p.u., respectively.

A photograph of the laboratory hardware setup reflecting the conceptual HIL architecture in Figure 1 is presented in Figure 14. We access the ADMS server that is located at a different place through its user interface software. Note that the ADMS server is placed in a different location as the HIL setup, and we access the ADMS server through its user interface software. The elements on the left in Figure 14 are the controller/device under test, including CHIL testing of the ADMS server, the Heila coordinator, and two Heila local controllers and PHIL testing of two DER racks with six hardware devices connected at two separate PCCs. We simulated 161 local controllers running in the laptop as the software controller under test.

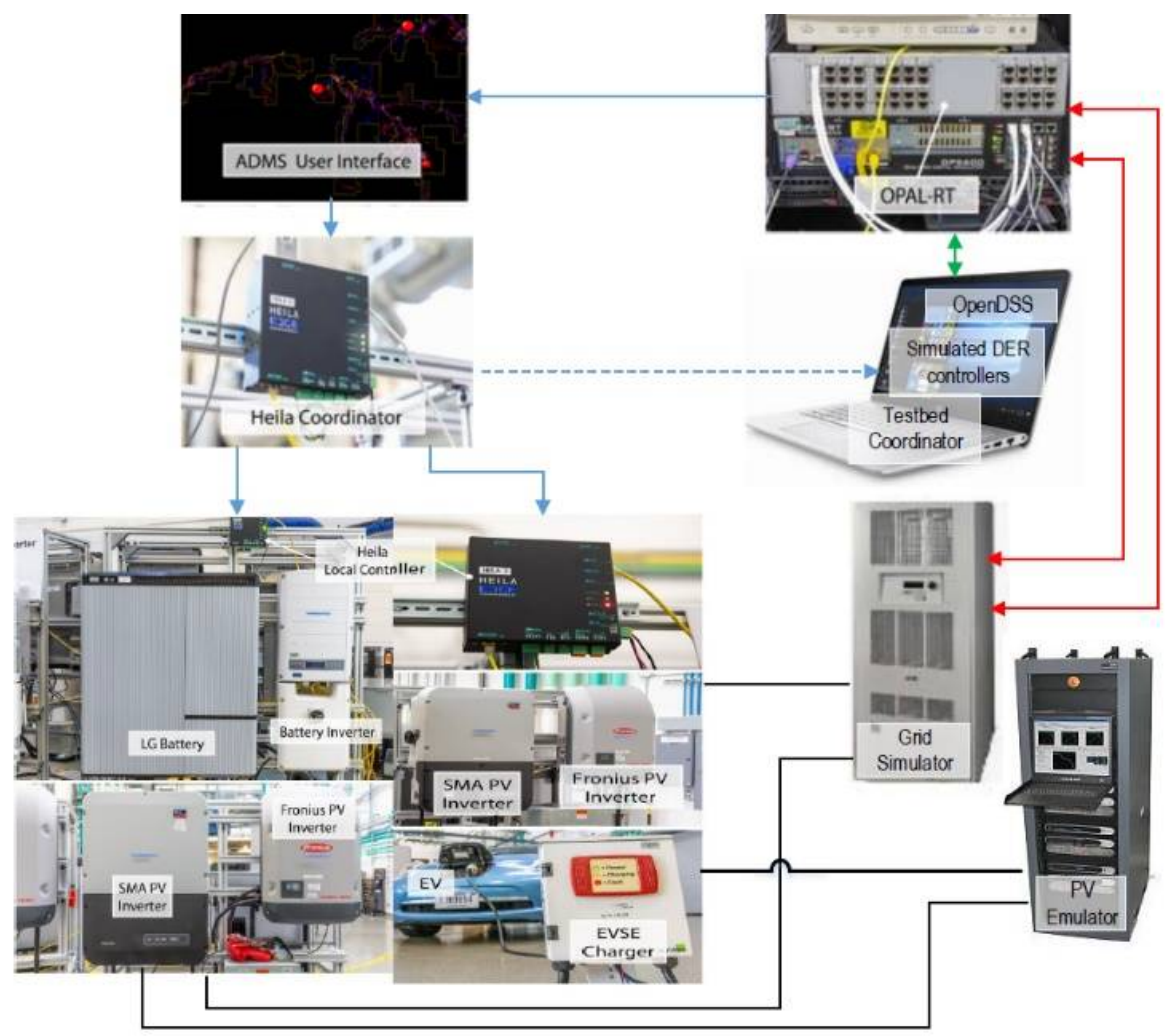

Figure 14. Components of the experimental setup of the HIL platform. Photos by NREL. 
In this test, the time steps in the Heila coordinator and Heila local controllers are $5 \mathrm{~s}$ and $2 \mathrm{~s}$, respectively. Representative results reflect that the HIL platform works collectively, and the DERMS control algorithms function correctly. The coordinated control across the ADMS, DERMS and DERs are presented in Figures 15-19.

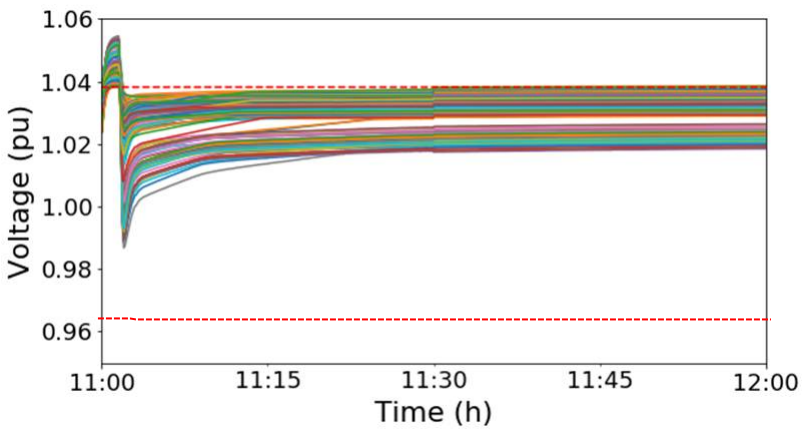

(a)

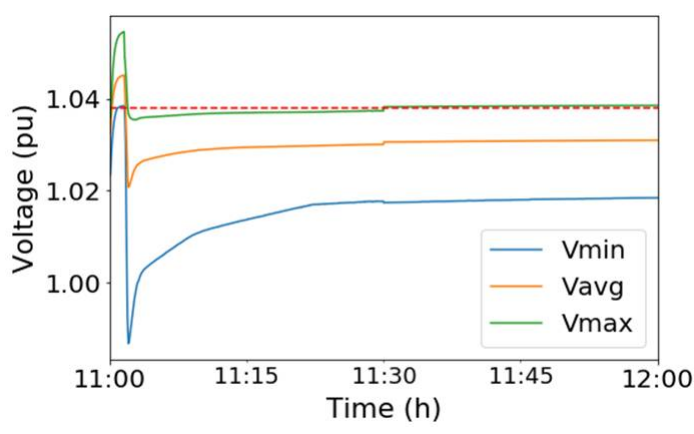

(b)

Figure 15. Voltage measurements of the scenario with DERMS control: (a) all the voltages across the feeder and (b) the average, maximal, and minimal voltages.

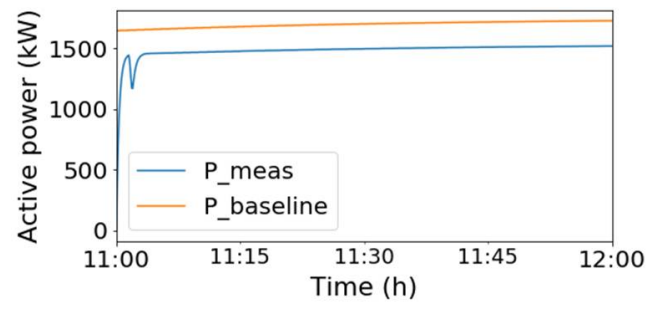

(a)

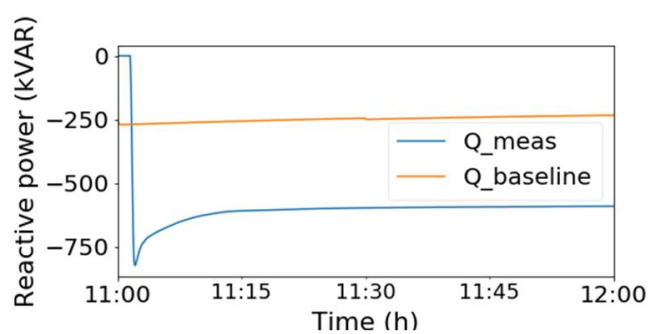

(b)

Figure 16. Total PV measurements: (a) active power output in baseline mode (P_baseline) and active power output with DERMS control (P_meas) and (b) reactive power output in baseline mode (Q_baseline) and reactive power output with DERMS control (Q_meas).

Figure 15 shows the voltage measurements of the scenario with DERMS control. At the beginning of the simulation, the voltages exhibit large transients because of the dynamics caused by the PV controlled by the DERMS. A closer look shows that all the voltages start to move toward steady-state approximately $2 \mathrm{~min}$ after reaching the peak point. This is because with the feedback control-the error cancellation and the gradient descent effect- the voltages gradually move toward the target values. As shown by the results, all the voltages are regulated under the upper limit with increasing solar irradiance and increasing PV generation. These results demonstrate the fast and smooth dynamics of the distribution system voltages and the satisfactory performance of using the DERMS for voltage regulation.

Figure 16 shows the total PV measurements with DERMS control and with volt/VAR autonomous mode (baseline). Compared to the baseline scenario, there is some curtailment of active power. In the baseline scenario, approximately $250 \mathrm{kVar}$ of reactive power is absorbed by all the PV inverters, and the active power of the PV inverters is barely curtailed because the reactive power is very small, which allows the active power to still output the available power. For the case with DERMS control, the calculated power curtailment is $12.73 \%$, which allows room for the reactive power to contribute to voltage regulation with approximately $625 \mathrm{kVar}$ reactive power absorbed by PV. The response of the PV in the baseline scenario shows that volt/VAR autonomous mode results in smooth dynamics in the PV. Under this high-voltage scenario, the DERMS control has a "brutal" force that pushes all the controlled PV to operate in the direction to curtail active power and increase reactive power absorption. This results in the output active and reactive power of all PV. 

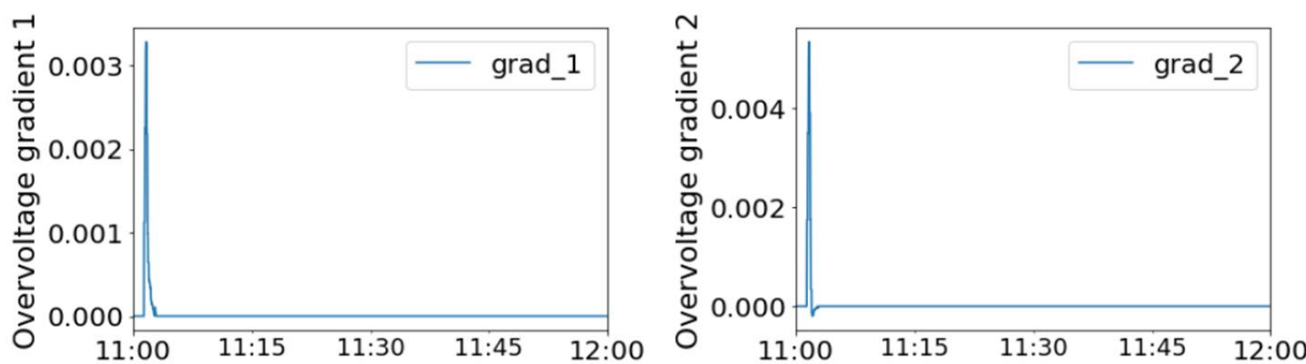

(a)
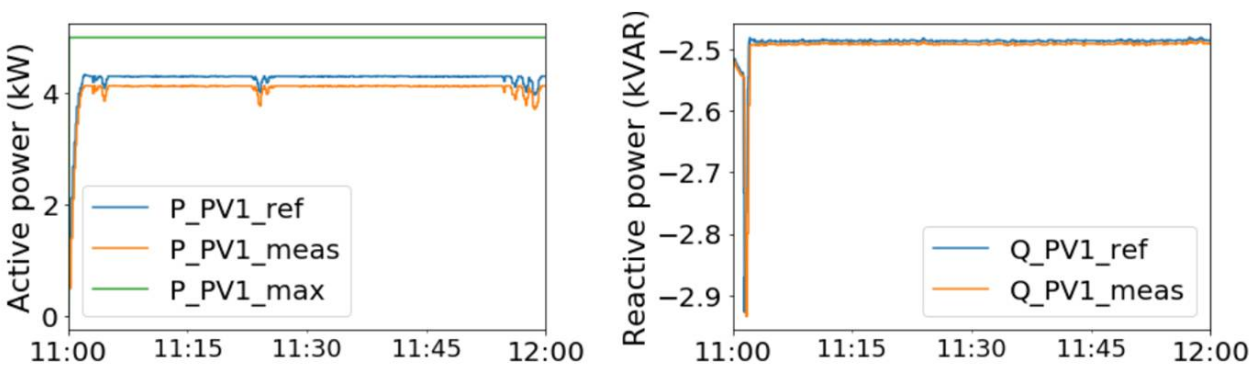

(b)
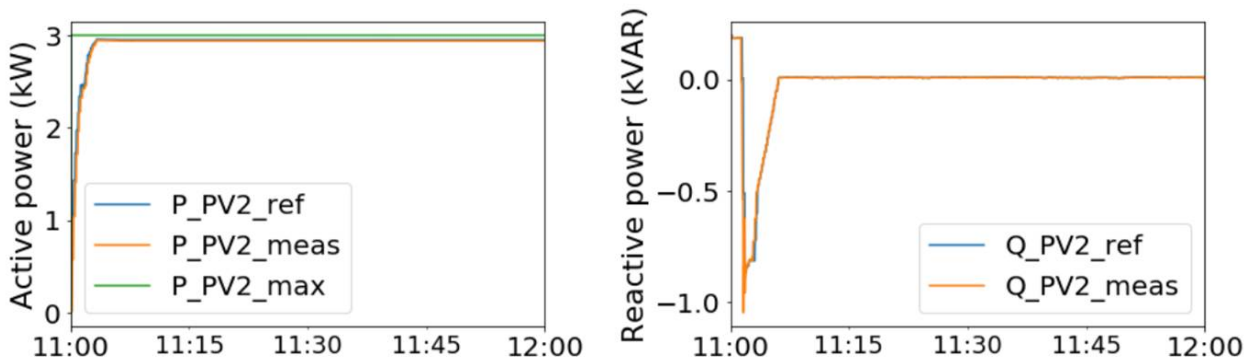

(c)
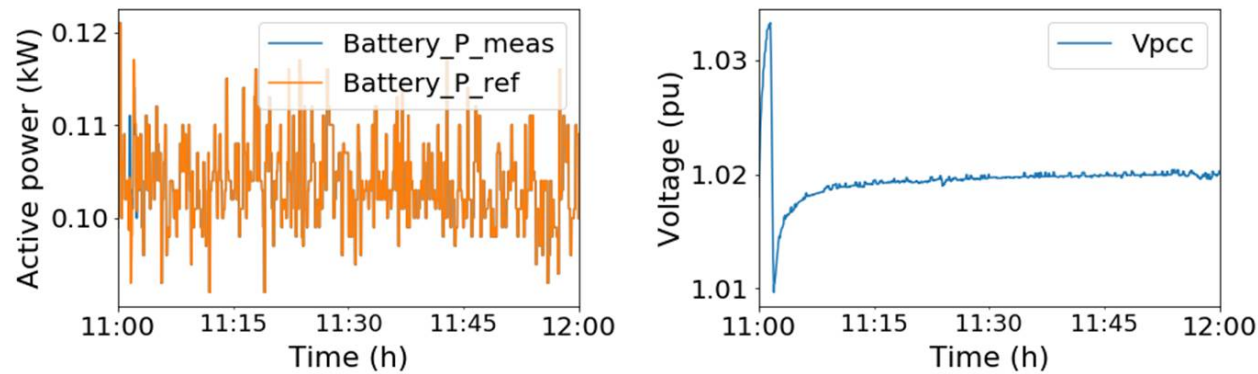

(d)

Figure 17. Results of hardware Heila DER local controller for Rack 1: (a) the dual variables received from the Heila coordinator; (b) the optimal power set points and measurements of PV inverter 1; (c) the optimal power set points and measurements of PV inverter 2 and (d) the optimal power set points and measurements of the battery inverter (left) and the PCC voltage of Rack 1 (right). 

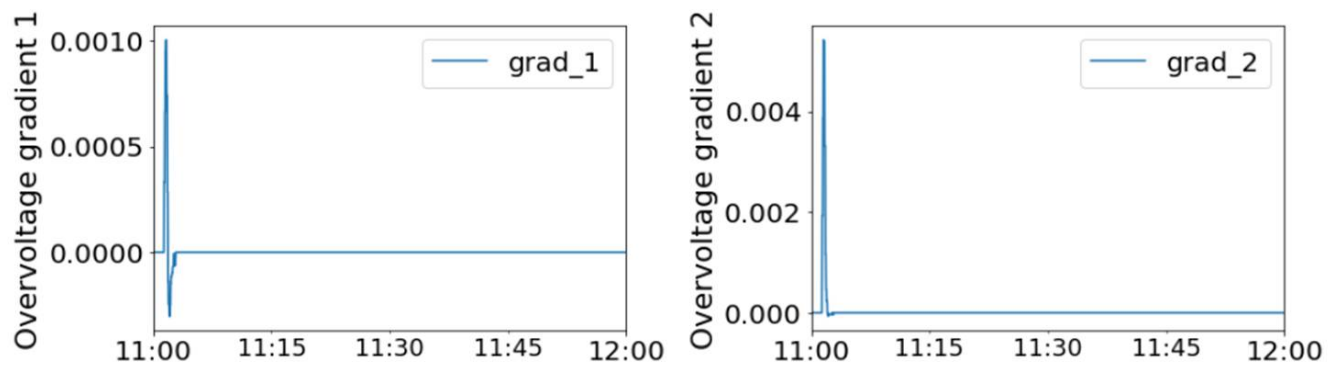

(a)
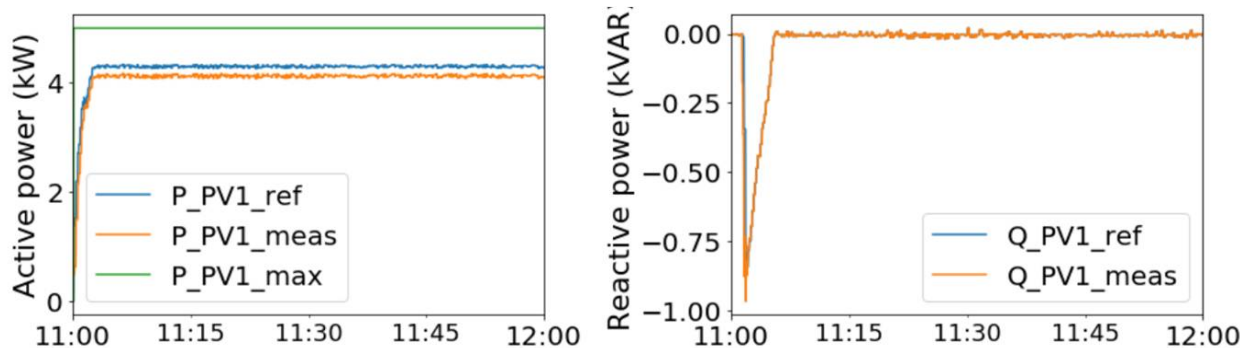

(b)
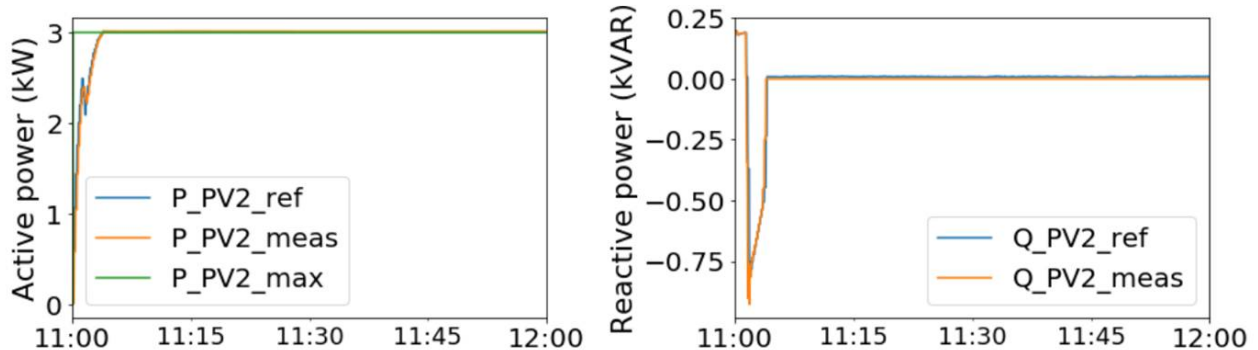

(c)
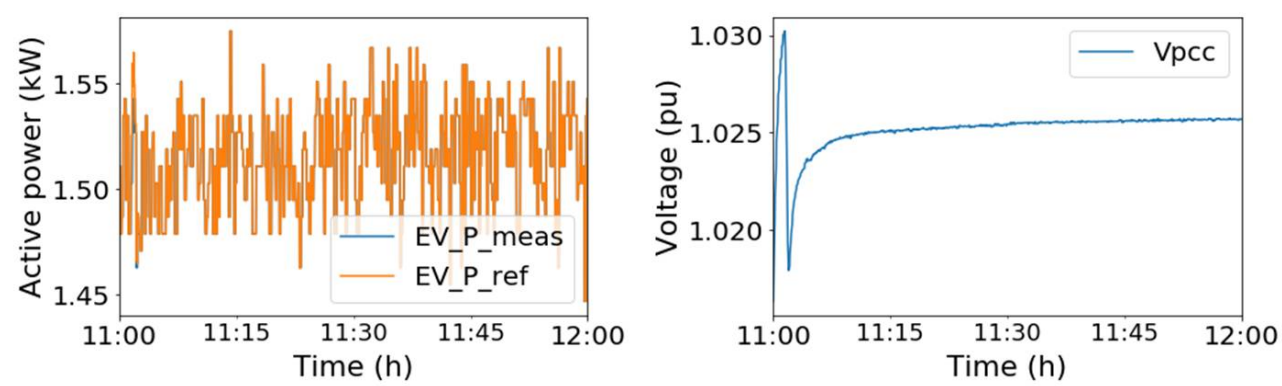

(d)

Figure 18. Results of hardware Heila DER local controller for Rack 2: (a) the dual variables received from the Heila coordinator; (b) the optimal power set points and measurements of PV inverter 1; (c) the optimal power set points and measurements of PV inverter 2 and (d) the optimal power set points and measurements of the EV (left) and the PCC voltage of Rack 2 (right). 

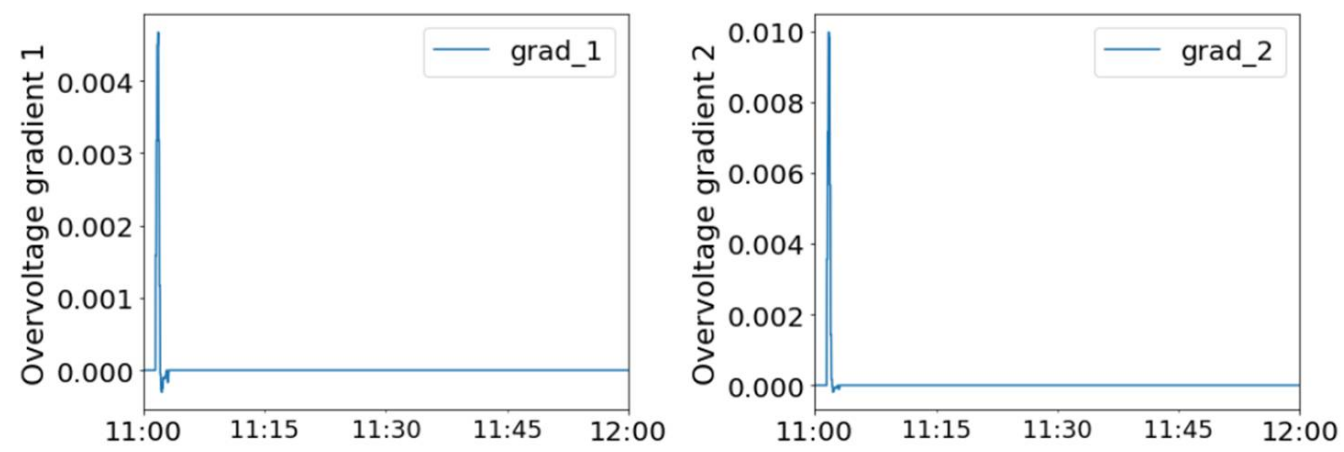

(a)
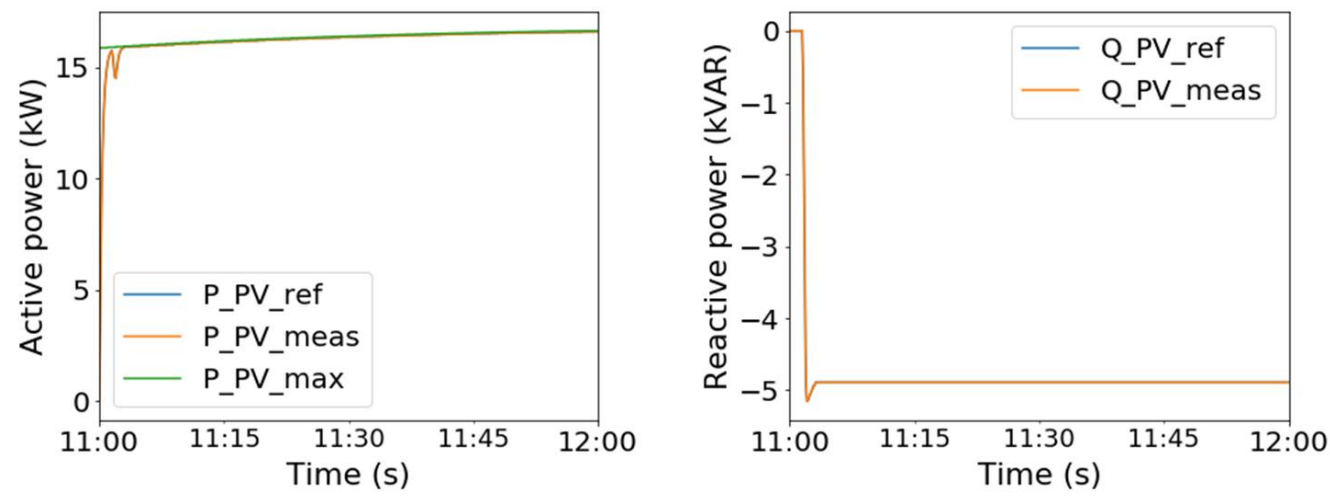

(b)

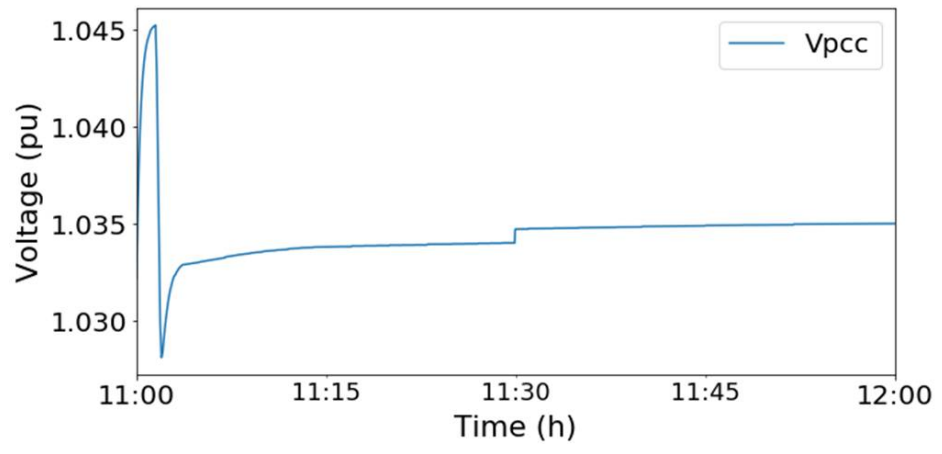

(c)

Figure 19. Results of a selected simulated local controller of PV: (a) the dual variables received from the Heila coordinator; (b) the optimal power set points and measurements of PV and (c) the PCC voltage of PV.

The results of the hardware Heila DER local controller for Rack 1 are presented in Figure 17. The top trace shows the dual variables of the hardware DERs received from the Heila controller, which exhibit large overshoots in both variables and a small undershoot in the second variable. A closer look shows that the two dual variables reach steady-state near 11:04 a.m., 4 min after starting the test. Then, they are kept at zero during the rest of the test because all the voltages are maintained within the limits. Figure $17 \mathrm{~b}-\mathrm{d}$ shows the responses of each hardware inverter, the two PV inverters (PV 1 and PV 2), and one battery inverter. Each inverter can follow the power setpoint closely. Even though the hardware inverters are connected at the same location, the responses of the inverters differ from each other. For instance, PV 1 has small ripples in the active power output, and the reactive power output reaches a steady state of absorbing $2.5 \mathrm{kVar}$ reactive power and PV 2 exhibits smooth transients in the active power output during the test and outputs zero VAR reactive power in steady-state. The differences in the two PV inverters' responses are caused by the differences in the capacities, with PV 1 at $5 \mathrm{kVA}$ and PV 2 at $3 \mathrm{kVA}$. The battery inverter is 
controlled to discharge a small amount of active power-approximately $105 \mathrm{~W}$-because of the high state of charge $(82 \%)$ during the test. The PCC voltage where the hardware inverters are connected exhibits some ripples, which are caused by harmonics of the output voltages and currents from the real hardware inverters.

The results of the hardware Heila DER local controller for Rack 2 are presented in Figure 18. The top trace shows the dual variables of the hardware DERs received from the Heila controller, which exhibit large overshoots in the two variables and a small undershoot in the first variable. These two variables reach a steady state near 11:04 a.m. Responses of the two PV inverters are presented in Figure 18b,c, which shows that both PV inverters track their power setpoints well. PV 1 curtails active power during the test and absorbs reactive power to regulate the voltage in the beginning and stops absorbing reactive power in steady-state and PV 2 has no curtailment in the active power and shows a response similar to PV 1 in the reactive power. Note that PV 2 has smoother active and reactive power outputs than PV 1 because the two inverters are from different PV manufactures. The EV is controlled to charge $1.5 \mathrm{~kW}$ of active power $(22.7 \%$ of the full capacity) to maintain the system voltage within the limits. The PCC voltage is maintained within the limits, and it shows small ripples caused by the harmonics from the hardware inverters.

One of the 161 simulated PV inverters with high PCC voltage is selected to show the responses of the simulated PV and the control performance of the DERMS. As shown in Figure 2, the Heila coordinator sends the optimization parameters (dual variables) to the simulated DER local controllers via HTTP link, and the simulated local controllers output the optimal power set points and send them to the simulated PV in OpenDSS through the testbed coordinator. The dual variables received by the simulated PV inverter are presented in Figure 19a. Because the dual variables are related to the upper voltage violations, each dual variable is larger and has a higher PCC voltage than those presented in Figures 17 and 18. The active and reactive power output tracks the power reference very well. The dynamic response of the active power is similar to the real hardware PV inverters. The reactive power has a smaller undershoot, and it outputs a large amount steadily and reaches a steady-state smoothly. Both active and reactive power exhibit faster dynamic responses than the hardware, which is expected because there are fewer delays involved with simulated PV inverters. The PCC voltage of the simulated PV shows that the voltage is regulated within target operating limits.

\section{Discussion}

This example HIL platform demonstrates the feasibility and efficacy of evaluating DERMS systems. Voltage regulation is the performance we evaluate. This platform can be expanded for more test scenarios/conditions. A few directions are summarized as follows:

- Evaluate futuristic scenarios with higher renewable penetration (e.g., 100\% renewables)

- Evaluate other functionalities of DERMS, such as VPP, peak load management/shaving

- Evaluate coordinated voltage regulation between ADMS and DERMS with ADMS regulating legacy devices (e.g., capacitor bank, LTC and voltage regulators) and DERMS regulates fast-response DERs

- Test more hardware inverters from various vendors with an easy plug-and-play feature of the platform

- Test communication systems, such as loss of communication, package loss, and new communication protocols of an inverter (e.g., 2030.5, SunSpec ModBus, and OpenADR)

\section{Conclusions}

This paper presented the performance evaluation of coordinated voltage regulation across ADMS, DERMS, and DERs using an advanced HIL platform. This HIL platform was used as an example to demonstrate the feasibility of a proposed generic platform, which includes the HIL platform (digital real-time simulator and power amplifiers) and the systems under test (ADMS, DERMS, DER inverters). We presented the implementation details of this example HIL platform, including co-simulation of the utility 
distribution feeder in real-time, the communications and gateway in the ADMS, the communications and control algorithms in the DERMS hardware controllers (Heila coordinator and Heila local controllers), and the closed-loop PHIL testing of six hardware DER inverters. Special interest was in the time step in the DERMS hardware controllers, and we investigated the voltage regulation performance under different time steps in the DERMS coordinator and local controllers. The results show that the time step in the DERMS coordinator influences the control performance more than the time step in the DERMS local controllers. Finally, an integrated test was performed in the peak solar time period to demonstrate the superior voltage regulation performance with DERMS control against the baseline mode with all PV inverters operating in autonomous volt-VAR mode. The results also illustrate that: the dual variables of the local controllers indicate that the DERMS algorithm converges, responds to system voltages and regulates system voltages with fast dynamics; (2) the hardware PV inverters in the same rack have similar responses in active power, while the reactive power responses are different; (3) battery inverter does not play a big role in voltage regulation, and EV is dispatched only $22.7 \%$ of full power for voltage regulation; (4) the harmonics and noise in real inverters are replicated by the simulated controlled source in OPAL-RT and the terminal voltage exhibits some harmonics, which shows the advantages of testing real hardware inverters and (5) the simulated PV inverter behaves similarly to the hardware PV inverter 1 in DER rack 1, which further indicates the hardware inverters work correctly. The main limitation of this example platform is that the simulated DER local controllers in the HELICS framework cannot function exactly the same as the real hardware controller because they are only software code without communication and operation systems as in the real controller. This will make the DERMS function in an ideal situation without any communication issues that might happen in real life.

This platform provides a realistic laboratory testing environment, and the testing results provide valuable insights for the utility prior to field deployment, including where to place the meters and the data rate in the ADMS SCADA; whether the DERMS functions in the field; the expected responses of the DERs and how the DERMS and ADMS coordinate with each other. For future DERMS-related projects, the co-simulation can be used to simulate distribution systems for different utility partners, software reconfiguration can be made in a real-time digital simulator for the grid simulator, the elements in the system under test can be changed to test products from different vendors with reconfigured communications and functions, and the DER hardware inverters can be dispatched by different external controllers. Therefore, the generic framework shown in Figure 1 provides great flexibility and extension ability for testing various DERMS technologies in a realistic laboratory testing environment.

Author Contributions: J.W.: Develop the conceptual framework of HIL evaluation, perform the HIL testing, process and analyze data, write the original draft. H.P.: help develop the co-simulation, performs baseline study, and support the HIL testing. F.D.: project administration, discussion, and formal analysis. M.B.: conceptualization and supervision. M.S.-D.: conceptualization and supervision. All authors have read and agreed to the published version of the manuscript.

Funding: This research is funded by U.S. Department of Energy Office of Energy Efficiency and Renewable Energy Office of Electricity Advanced Grid Research program; the U.S. Department of Energy Advanced Research Projects Agency-Energy (ARPA-E) NODES program and Holy Cross Energy under a cooperative research and development agreement (CRD-18-756).

Acknowledgments: This work was supported by Alliance for Sustainable Energy, LLC, the manager and operator of the National Renewable Energy Laboratory for the U.S. Department of Energy (DOE) under Contract No. DE-AC36-08GO28308. This work was performed in NREL's Energy System Integration Facility (ESIF).The views expressed in the article do not necessarily represent the views of the DOE or the U.S. Government. The U.S. Government retains and the publisher, by accepting the article for publication, acknowledges that the U.S. Government retains a nonexclusive, paid-up, irrevocable, worldwide license to publish or reproduce the published form of this work, or allow 
others to do so, for U.S. Government purposes. The authors would like to thank Seth Dew from Heila for his help configuring the Heila coordinator and local controllers for multiple time step testing.

Conflicts of Interest: The authors declare no conflict of interest.

\section{References}

1. Cook, J.J.; Ardani, K.B.; O'Shaughnessy, E.J.; Margolis, R.M.; Smith, B. Expanding PV Value: Lessons Learned from Utility-Led Distributed Energy Resource Aggregation in United States; Technical Report; National Renewable Energy Laboratory: Golden, CO, USA, 2018. Available online: https:/ / www.nrel.gov/docs/fy19osti/71984.pdf (accessed on 1 October 2021).

2. Jan, V. Navigant Energy, Distributed Energy Resources: Lead to Follow. 2015. Available online: https://www.aspeninstitute.org/ wp-content/uploads/files/content/images/2015\%20Aspen \%20Institute $\% 20$ Policy $\% 20$ Form $\% 20$ Presentation $\% 20$ Navigant $\%$ 20Jan\%20Vrins\%20FINAL\%20UPDATED.pdf (accessed on 1 October 2021).

3. Microgrid Knowledge, What Are Distributed Energy Management Systems? 2018. Available online: https://microgridknowledge. com/derms-next-generation-grid/ (accessed on 1 October 2021).

4. Seal, B.; Renjit, A.; Deaver, B. Understanding DERMS; Electric Power Research Institute: Palo Alto, CA, USA, 2018; Available online: https://www.epri.com/\#/pages/product/000000003002013049/?lang=en (accessed on 1 October 2021).

5. Strezoski, L.; Stefani, I.; Brbaklic, B. Active Management of Distribution Systems with High Penetration of Distributed Energy Resources. In Proceedings of the IEEE EUROCON 2019-18th International Conference on Smart Technologies, Novi Sad, Serbia, 1-4 July 2019.

6. Zhou, X.; Liu, Z.; Zhao, C.; Chen, L. Accelerated Voltage Regulation in Multi-Phase Distribution Networks Based on Hierarchical Distributed Algorithm. IEEE Tran. Power Syst. 2020, 35, 2047-2058. [CrossRef]

7. Fletcher, F. AMI Enabled Energy Demand Management with Distributed Energy Resources. In Proceedings of the IEEE PES T\&D 2010, New Orleans, LA, USA, 19-22 April 2010.

8. Aki, H.; Wakui, T. Optimal Management of Fuel Cells in a Residential Area by Integrated-Distributed Energy Management System (IDEMS). In Proceedings of the IEEE Power \& Energy Society Innovative Smart Grid Technologies Conference (ISGT), Minneapolis, MN, USA, 6-9 September 2016.

9. Tushar, W.; Chai, B.; Yuen, C.; Smith, D.B.; Wood, K.L.; Yang, Z.; Poor, H.V. Three-Party Energy Management with Distributed Energy Resources in Smart Grid. IEEE Tran. Ind. Electron. 2015, 62, 2487-2498. [CrossRef]

10. Saad, A.; Youssef, T.; Elsayed, A.T.; Amin, A.; Abdalla, O.H.; Mohammed, O. Data-Centric Hierarchical Distributed Model Predictive Control for Smart Grid Energy Management. IEEE Tran. Ind. Inform. 2019, 15, 4086-4098. [CrossRef]

11. Zehir, M.A.; Bagriyanik, M. Smart Energy Aggregation Network (SEAN): An Advanced Management System for Using Distributed Energy Resources in Virtual Power Plant Applications. In Proceedings of the 3rd International Istanbul Smart Grid Congress and Fair (ICSG), Istanbul, Turkey, 29-30 April 2015.

12. Nezamabadi, P.; Gharehpetian, G.B. Electrical Energy Management of Virtual Power Plants in Distribution Networks with Renewable Energy Resources and Energy Storage Systems. In Proceedings of the 16th Electrical Power Distribution Conference, Bandar Abbas, Iran, 19-20 April 2011.

13. Liang, Z.; Chen, H.; Wang, X.; Chen, S.; Zhang, C. Risk-Based Uncertainty Set Optimizaion Metohd for Energy Management of Hybrid AC/DC Microgrids with Uncertian Renewable Generation. IEEE Tran. Smart Grid 2020, 11, 1526-1542. [CrossRef]

14. Wang, J.; Zhao, C.; Pratt, A.; Baggu, M. Design of an Advanced Energy Management System for Microgrid Control Using a State Machine. Appl. Energy 2018, 228, 2407-2421. [CrossRef]

15. Wang, J.; Song, Y.; Li, W.; Guo, J.; Monti, A. Development of a Universal Platform for Hardware In-the-Loop Testing of Microgrids. IEEE Tran. Ind. Inform. 2014, 10, 2154-2165. [CrossRef]

16. Pratt, A.; Baggu, M.; Ding, F.; Veda, S.; Mendoza, I.; Lightner, E. A Test Bed to Evaluate Advanced Distribution Management Systems for Modern Power Systems. In Proceedings of the IEEE EUROCON 2019-18th International Conference on Smart Technologies, Novi Sad, Serbia, 1-4 July 2019; Available online: http:/ / eurocon2019.org/program.html?r (accessed on 1 October 2021).

17. Wang, J.; Blonsky, M.; Ding, F.; Drew, S.C.; Padullaparti, H.; Ghosh, S.; Mendoza, I.; Tiwari, S.; Martinez, J.E.; Dahdah, J.J.; et al. Performance Evaluation of Distributed Energy Resource Management via Advanced Hardware-in-the-Loop Simulation. In Proceedings of the IEEE Conference on Innovative Smart Grid Technologies (ISGT), Washington, DC, USA, 17-20 February 2020.

18. Paudyal, P.; Ding, F.; Ghosh, S.; Baggu, M.; Symko-Davies, M.; Bilby, C.; Hannegan, B. The Impact of Behind-the-Meter Heterogeneous Distributed Energy Resources on Distribution Grids. In Proceedings of the 47th IEEE Photovoltaic Specialists Conference (PVSC47), Calgary, AB, Canada, 14-19 June 2020.

19. Palmintier, B.; Krishnamurthy, D.; Top, P.; Smith, S.; Daily, J.; Fuller, J. Design of the HELICS high-performance transmissiondistribution-communication-market co-simulation framework. In Proceedings of the Workshop on Modeling and Simulation of Cyber-Physical Energy Systems (MSCPES), Pittsburgh, PA, USA, 18-21 April 2017; pp. 1-6.

20. Dall'Anese, E.; Simonetto, A. Optimal power flow pursuit. IEEE Trans. Smart Grid 2018, 9, 942-952. [CrossRef]

21. OPAL-RT, Software-in-the-Loop: Fastest Production Source Code Validation. Available online: https://www.opal-rt.com/ software-in-the-loop/ (accessed on 1 October 2021). 
22. Development of Application Function Blocks for Power-Hardware-in-the-Loop Interface Algorithm for Testing Grid-Connected Inverters. In Proceedings of the IEEE 9th International Symposium on Power Electronics for Distributed Generation Systems, Charlotte, NC, USA, 25-28 June 2018.

23. Bryan, P.; Julieta, G.; Kenny, G.; Peter, G.; Adarsh, N.; Tom, H.; Bruce, B.; Murali, B.; Jesse, G.; Ethan, B. Feeder Voltage Regulation with High-Penetration PV Using Advanced Inverters and a Distribution Management System, A Duke Energy Case Study; Technical Report; NREL/TP-5D00-65551; 2016. Available online: https:/ /www.osti.gov/biblio/1331479/ (accessed on 1 October 2021). 